\title{
Star formation histories of dwarf galaxies in the FIRE simulations: dependence on mass and Local Group environment
}

\author{
Shea Garrison-Kimmel ${ }^{\oplus},{ }^{1 \star} \dagger$ Andrew Wetzel ${ }^{\oplus},{ }^{2}$ Philip F. Hopkins ${ }^{\oplus},{ }^{1}$ \\ Robyn Sanderson ${ }^{\odot}, 1,3,4$ Kareem El-Badry ${ }^{\odot}, 5$ Andrew Graus, ${ }^{6,7}$ T. K. Chan, ${ }^{8}$ \\ Robert Feldmann ${ }^{\odot},{ }^{9}$ Michael Boylan-Kolchin ${ }^{\oplus},{ }^{7}$ Christopher C. Hayward, ${ }^{4}$ \\ James S. Bullock, ${ }^{6}$ Alex Fitts ${ }^{\oplus},{ }^{7}$ Jenna Samuel ${ }^{\oplus},{ }^{2}$ Coral Wheeler, ${ }^{1}$ Dušan Kereš ${ }^{8}$ \\ and Claude-André Faucher-Giguère ${ }^{\oplus 10}$ \\ ${ }^{1}$ TAPIR, Mailcode 350-17, California Institute of Technology, Pasadena, CA 91125, USA \\ ${ }^{2}$ Department of Physics, University of California, Davis, CA 95616, USA \\ ${ }^{3}$ Department of Physics \& Astronomy, University of Pennsylvania, 209 South 33rd St., Philadelphia, PA 19104, USA \\ ${ }^{4}$ Center for Computational Astrophysics, Flatiron Institute, 162 5th Ave., New York, NY 10010, USA \\ ${ }^{5}$ Department of Astronomy and Theoretical Astrophysics Center, University of California Berkeley, Berkeley, CA 94720, USA \\ ${ }^{6}$ Center for Cosmology, Department of Physics and Astronomy, University of California, Irvine, CA 92697, USA \\ ${ }^{7}$ Department of Astronomy, The University of Texas at Austin, 2515 Speedway, Stop C1400, Austin, TX 78712, USA \\ ${ }^{8}$ Department of Physics, Center for Astrophysics and Space Science, University of California at San Diego, 9500 Gilman Drive, La Jolla, CA 92093, USA \\ ${ }^{9}$ Institute for Computational Science, University of Zurich, CH-8057 Zurich, Switzerland \\ ${ }^{10}$ Department of Physics and Astronomy and CIERA, Northwestern University, 2145 Sheridan Road, Evanston, IL 60208, USA
}

Accepted 2019 September 4. Received 2019 July 19; in original form 2019 March 26

\begin{abstract}
We study star formation histories (SFHs) of 500 dwarf galaxies (stellar mass $M_{*}=$ $10^{5}-10^{9} \mathrm{M}_{\odot}$ ) from FIRE-2 cosmological zoom-in simulations. We compare dwarfs around individual Milky Way (MW)-mass galaxies, dwarfs in Local Group (LG)-like environments, and true field (i.e. isolated) dwarf galaxies. We reproduce observed trends wherein higher mass dwarfs quench later (if at all), regardless of environment. We also identify differences between the environments, both in terms of 'satellite versus central' and 'LG versus individual MW versus isolated dwarf central.' Around the individual MW-mass hosts, we recover the result expected from environmental quenching: central galaxies in the 'near field' have more extended SFHs than their satellite counterparts, with the former more closely resemble isolated (true field) dwarfs (though near-field centrals are still somewhat earlier forming). However, this difference is muted in the LG-like environments, where both near-field centrals and satellites have similar SFHs, which resemble satellites of single MW-mass hosts. This distinction is strongest for $M_{*}=10^{6}-10^{7} \mathrm{M}_{\odot}$ but exists at other masses. Our results suggest that the paired halo nature of the LG may regulate star formation in dwarf galaxies even beyond the virial radii of the MW and Andromeda. Caution is needed when comparing zoom-in simulations targeting isolated dwarf galaxies against observed dwarf galaxies in the LG.
\end{abstract}

Key words: galaxies: dwarf-galaxies: formation-Local Group-cosmology: theory.

\section{INTRODUCTION}

The star formation history (SFH) of a dwarf galaxy is one of its fundamental properties. It has implications for the $z=0$ dark matter density profile at fixed mass, as late-time star formation appears

^E-mail: sheagk@caltech.edu

$\dagger$ Einstein Fellow. to correlate with core formation (e.g. Oñorbe et al. 2015; Read, Agertz \& Collins 2016; Read, Walker \& Steger 2019). Dwarf SFHs further inform how interactions with the Milky Way (MW) impact satellites, either through comparisons with infall times inferred for individual satellites (e.g. Rocha, Peter \& Bullock 2012; Fillingham et al. 2019) or more broadly in comparing typical satellite SFHs with those of central (non-satellite) galaxies, as suggested by Brooks \& Zolotov (2014). They can also yield unique constraints on the contribution of dwarf galaxies to the reionizing background (e.g. 
Ricotti, Gnedin \& Shull 2008; Weisz, Johnson \& Conroy 2014c; Boylan-Kolchin et al. 2015). Moreover, the shape of a dwarf's SFH may be correlated with the growth of the halo at low halo masses $\left(10^{10} \mathrm{M}_{\odot}\right.$; e.g. Fitts et al. 2017) and with the kinematics of the gas and stars in the galaxy at slightly higher masses $\left(10^{11} \mathrm{M}_{\odot}\right.$; e.g. El-Badry et al. 2018b). Dwarf SFHs may even inform the nature of dark matter, as different DM models predict different accretion histories (e.g. Colín et al. 2015; Governato et al. 2015; Lovell et al. 2017; Bozek et al. 2019).

Observations have begun to provide detailed constraints on the SFHs of a large fraction of the dwarf galaxies in the Local Group (LG; defined as the cosmological volume containing the MW, M31, and all dwarf galaxies within $1 \mathrm{Mpc}$ of either of these hosts), typically by resolving the oldest main-sequence turn-off stars with space-based photometry. Weisz et al. (2014a) presented the largest such sample, with SFHs for 40 LG dwarf galaxies uniformly derived from HST observations (and also see Cole et al. 2007, 2014; Skillman et al. 2017). They found that higher mass galaxies form a higher fraction of their stars at later times, and that the central galaxies in the so-called Local Field (i.e. more than $300 \mathrm{kpc}$ from both the MW or M31, but still within the LG) typically form later than their satellite counterparts. Weisz et al. (2014b) argued that only two of those 40 dwarf galaxies are consistent with their star formation being completely halted (quenched) by reionization, though Brown et al. (2014) used similar observations of lower mass ultrafaint dwarf galaxies (stellar mass $M_{*} \lesssim 3 \times 10^{4} \mathrm{M}_{\odot}$ ) to argue that reionization becomes increasingly important at lower masses, as all six of the galaxies in their sample stop forming stars by $z \simeq 2$. Rodriguez Wimberly et al. (2019) further argued that environmental effects caused by the hot halo of the MW (e.g. Gupta et al. 2012) including ram pressure and turbulent viscous stripping (Gunn \& Gott 1972; Nulsen 1982; Hester 2006; Fillingham et al. 2016), which actively remove gas from satellite galaxies, and 'starvation,' where the accretion of fresh gas is suppressed (Larson, Tinsley \& Caldwell 1980; Kawata \& Mulchaey 2008) - cannot reproduce such early quenching times, strengthening the case that the UV background is responsible. ${ }^{1}$

At higher masses, however, it appears that environment is more important in shaping dwarf SFHs, as epitomized by the simple observation that the majority of satellite dwarfs have no detectable $\mathrm{H}$ I while the majority of centrals do (e.g. Einasto et al. 1974; McConnachie 2012; Spekkens et al. 2014). The concept of environmental quenching is supported by the results of Geha et al. (2012), who showed that the fraction of quenched $M_{*}=$ $10^{8}-10^{9} \mathrm{M}_{\odot}$ galaxies is consistent with zero for dwarfs $\gtrsim 1 \mathrm{Mpc}$ from the nearest MW-mass (or larger) host, but rises sharply at smaller distances. Similarly, Gallart et al. (2015) argued that dwarf galaxies in the LG can be grouped into 'fast' and 'slow' rising SFHs, where the former have positions and/or velocities consistent with previous interactions with the MW or M31. Several authors have used statistical arguments to show that the preponderance of quenched satellites around the MW suggests that quenching times must be quite short at low masses $\left(\lesssim 2\right.$ Gyr for $\left.M_{*} \lesssim 10^{8} \mathrm{M}_{\odot}\right)$, and longer ( $\gtrsim 6 \mathrm{Gyr}$ ) at higher masses, provided that quenching is directly linked with entering the virialized volume of the MW (e.g. Wetzel et al. 2013; Wheeler et al. 2014; Fillingham et al. 2015; Wetzel, Tollerud \& Weisz 2015). Fillingham et al. (2018) recently

\footnotetext{
${ }^{1}$ Also see Bland-Hawthorn, Sutherland \& Webster (2015), who argued that very low-mass haloes (possibly as small as $10^{7} \mathrm{M}_{\odot}$ ) could form stars prior to reionization.
}

used a similar technique to argue that quenching processes that operate purely within the virial radius of the hosts are insufficient to explain the quenched galaxies at $\gtrsim 600 \mathrm{kpc}$ from the MW/M31, though dwarf-dwarf interactions may lead to more extended (and perhaps more slowly collapsing) $\mathrm{H}$ I reservoirs relative to isolated dwarf galaxies (Pearson et al. 2016). Emerick et al. (2016) used idealized wind-tunnel simulations to argue that stripping, aided by supernovae feedback, could not explain the short quenching timescales inferred by the above works even for satellites.

The question of satellite quenching is further complicated by the results of the Satellites Around Galactic Analogs (SAGA) Survey: 26 of the 27 satellites identified around the $8 \mathrm{MW}$-mass galaxies in their sample appear to be star forming, though the dwarf sample only reaches $M_{*} \gtrsim 10^{7} \mathrm{M}_{\odot}$ (Geha et al. 2017). In slight contrast, Tanaka et al. (2018) reached $M_{*} \gtrsim 10^{6} \mathrm{M}_{\odot}$ and identified a mix of blue and red satellite galaxies around two other MW analogues, with the authors classifying the majority of the blue (i.e. star forming) sample as 'possible' dwarf galaxies, rather than secure detections.

Meanwhile, as the typical resolutions of hydrodynamic cosmological simulations increase, authors have begun to compare simulated dwarf SFHs to the observations detailed above. Many of these works have focused on highly isolated (i.e. field) dwarf galaxies, which can be simulated at much higher resolutions than dwarfs around MW-mass hosts because the MW-mass galaxy itself dominates the run-time of the latter such simulations. For example, Fitts et al. (2017) presented dwarf SFHs taken from simulations using the FIRE ${ }^{2}$ (Feedback In Realistic Environments) physics. They demonstrated overall agreement with observations in terms of the range of dwarf SFHs. They further argued that the $z=0$ stellar mass scales with the maximum circular velocity $V_{\max }$ of the halo (at fixed halo mass), though they did not identify any clear trends with the shapes of the SFHs. Wright et al. (2019) used GASOLINE (Wadsley, Stadel \& Quinn 2004) simulations of similarly isolated dwarf galaxies to understand why the SFHs of some dwarfs 'reignite' after apparently quenching. They found that interactions with gaseous streams in the intergalactic medium can compress gas around the dwarf to the point where it begins to cool and form stars.

Other authors have explored the SFHs of dwarf galaxies that evolve around MW-mass hosts, though typically at lower resolutions than the works above. Benítez-Llambay et al. (2015) used the Constrained Local UniversE Simulations (CLUES; Gottloeber, Hoffman \& Yepes 2010), which target LG-like pairs, to examine the impact of reionization on central galaxies. They found great diversity in their simulated SFHs, and argued that 'gaps' in star formation at intermediate ages (cosmic time $t \simeq 4-8 \mathrm{Gyr}$ ) can be attributed to reionization. Similar to Fitts et al. (2017), they argued for the importance of $V_{\max }$ in haloes near the reionization suppression scale.

More recently, Wetzel et al. (2016) showed that the FIRE-2 prescriptions accurately reproduce the diversity in observed dwarf SFHs, the high fraction of quenched satellites near the MW at $z=$ 0 , and the general dependence on galaxy mass, though that paper examined only a single MW-mass host galaxy. Given the aforementioned results from SAGA and Tanaka et al. (2018), it is unclear if the high quenched fraction around the MW is representative of MW-mass satellite populations, at least for $M_{*} \gtrsim 10^{7} \mathrm{M}_{\odot}$.

Finally, Digby et al. (2019) also examined the SFHs of dwarf galaxies in the APOSTLE (Fattahi et al. 2016; Sawala et al. 2016) and AURIGA (Grand et al. 2017) simulations. They found that

\footnotetext{
${ }^{2}$ http://fire.northwestern.edu
} 
late-time ( $t \gtrsim 8 \mathrm{Gyr}$ ) star formation is suppressed in satellite galaxies relative to dwarf centrals of the same mass, and that low (high) mass dwarf centrals have SFHs that decline (rise) at late times. In a related work, Simpson et al. (2018) examined the gas content of dwarf galaxies in the AURIGA simulations. Dwarf galaxies in their simulations are susceptible to ram pressure stripping, such that those at smaller host distances are more likely to be quenched and gas poor. At their lowest masses $\left(M_{*} \simeq 10^{6} \mathrm{M}_{\odot}\right)$, all dwarf galaxies that are either satellites today or were satellites in the past are quenched, with the quenched fraction falling monotonically with increasing stellar mass.

Finally, Buck et al. (2019) studied the properties of dwarf galaxies around MW-sized hosts in the NIHAO simulations, separated by their distance to the MW analogue. They found that those dwarf SFHs roughly reproduce LG observations (though perhaps with a bias towards earlier star formation), and that the satellites and 'nearby' centrals (defined in that work as between $R_{200}$ and $2.5 \times$ $R_{200}$, or roughly $200 \lesssim d_{\text {host }} \lesssim 500 \mathrm{kpc}$ ) exhibit SFHs that are similar to the satellite galaxies with the clearest differences in gas content and late-time SFH occurring beyond $2.5 \times R_{200}$. The authors further argued that the environmental effects that truncate star formation also result in a lowered velocity dispersion at fixed $M_{*}$.

These results establish two primary questions, which we explore here: (1) Do the FIRE-2 physics, which accurately reproduce many other attributes of the LG dwarf galaxies, reproduce the observed trends with $M_{*}$ and environment over a statistical sample of dwarf galaxies? (2) How do the predicted SFHs vary between environments? For example, do simulations of highly isolated dwarf galaxies represent a fair comparison to centrals in the LG, and does the presence of a second MW-mass galaxy impact star formation in the dwarf galaxies throughout the LG? In this paper, we address these questions by analysing a large suite of dwarf galaxies simulated with identical physics in a variety of environments. We describe the simulations and our sample in Section 2, present and discuss our results (and caveats to those results) in Section 3, and summarize our conclusions in Section 4. All of our simulations adopt flat $\Lambda \mathrm{CDM}$ cosmologies with $h \simeq 0.7$ and $\Omega_{\mathrm{m}} \simeq 0.3$ (e.g. Larson et al. 2011; Planck Collaboration XIII 2016; Planck Collaboration VI 2018).

\section{SIMULATIONS}

All of our dwarf galaxies are taken from cosmological, hydrodynamic zoom-in (Katz \& White 1993; Oñorbe et al. 2014) simulations that are a part of the FIRE project (Hopkins et al. 2014) and run using the 'FIRE-2' version of the code presented in Hopkins et al. (2018b, i.e. with identical physics and code). All simulations were initialized with MUSIC (Hahn \& Abel 2011) and evolved with GIZMO (Hopkins 2015) ${ }^{3}$ in its meshless finite-mass (MFM) mode. The FIRE physics modules are described in detail in the papers above; briefly, we include radiative heating/cooling for 10 $10^{10} \mathrm{~K}$; allow for star formation in dense gas that is Jeans unstable, molecular and self-shielding (Krumholz \& Gnedin 2011), and selfgravitating (Hopkins, Narayanan \& Murray 2013); and include stellar feedback via radiation pressure, photoelectric heating and photoionization, supernovae Types Ia and II and metal mass loss assuming each star particle is a single stellar population with a Kroupa (2001) initial mass function. The simulations adopt the 2011 December update of the Faucher-Giguère et al. (2009) UV

${ }^{3}$ http://www.tapir.caltech.edu/ phopkins/Site/GIZMO.html background model, ${ }^{4}$ which was designed to produce a reionization optical depth consistent with WMAP-7, with a reionization redshift $z_{\text {reion }} \simeq 10$. Most of our simulations (all but those taken from Fitts et al. 2017) include turbulent metal diffusion (Hopkins 2017), which yields more realistic stellar metallicity distributions in simulated dwarf galaxies (Escala et al. 2018) but has a negligible effect on the star formation (Su et al. 2017 and Fig. A1).

We expect each of our feedback channels to impact (i.e. slow) star formation slightly differently. Many authors (e.g. Wheeler et al. 2018; Rodriguez Wimberly et al. 2019) have argued that the metagalactic UV background dominates the evolution of the lowest mass ultrafaint dwarf galaxies, with the dividing line typically set at $M_{*} \simeq 10^{5} \mathrm{M}_{\odot}$, the lower edge of our sample. At higher masses, internal stellar feedback channels dominate over the metagalactic background at roughly all times. As outlined in section 9 of Hopkins et al. (2018b), supernovae feedback is the most important for regulating star formation at roughly all masses. Radiation pressure, meanwhile, 'smooths' out star formation in dwarf galaxies; this typically results in more star formation overall (and particularly at late times) by de-clustering supernovae in time such that no single burst is strong enough to completely quench the dwarf galaxy (Hopkins et al. 2018a). Stellar winds also promote late-time star formation: without the gas provided by mass loss from AGB and OB stars, dwarf galaxies show suppressed SFRs past $t \simeq 4-6 \mathrm{Gyr}$.

We analyse dwarf galaxies from zoom-in simulations that target LG-like pairs of MW-mass hosts, isolated MW-mass hosts, and highly isolated dwarf central galaxies (i.e. without including any MW-mass hosts in the zoom-in volume). We plot our sample as a function of stellar mass, using decade-wide bins, and separated by environment in Fig. 1, and list the parent simulations for the full sample in Table 1, which gives the primary galaxy (or, in the case of the LG-like runs, galaxies) in each run along with their stellar and halo masses, a measure of their isolation, mass resolution, and the publication where each halo first appeared at the adopted resolution. ${ }^{5}$ We also direct the reader to El-Badry et al. (2018a,b), who analysed the $\mathrm{H}$ I properties of the majority of the isolated central sample; to Garrison-Kimmel et al. (2018), who analysed the morphologies and growth histories of the majority of the MW-mass hosts (though typically at lower resolution); and to Sanderson et al. (2018), who studied the mass in the stellar haloes of the MW-mass hosts (again typically at lower resolution).

In the LG and isolated MW simulations, we identify (sub)structure in the dark matter particles using the Rockstar (Behroozi, Wechsler \& Wu 2013) 6D halo finder, then use a similar (though not identical) process as Necib et al. (2018) to assign star particles to those overdensities. The method is described in detail in Samuel et al. (2019) but, in short, we select star particles that are located within the radius of the halo (as reported by Rockstar) and moving with a relative velocity that is within $2 \times V_{\max }$. We then define $R_{90}$ as the radius that encloses 90 per cent of that stellar mass and $\sigma_{*}$ as the velocity dispersion of those star particles. Finally, we iteratively remove stars that are $>1.5 \times R_{90}$ from either the galaxy or (sub)halo centre, or that have a velocity offset $>2 \times \sigma_{*}$, until

\footnotetext{
${ }^{4}$ Available at http://galaxies.northwestern.edu/uvb/.

${ }^{5}$ Our sample includes two new isolated MW-mass hosts, m12r and $\mathrm{m} 12 \mathrm{w}$, selected to contain Large Magellanic Cloud-like satellites in their darkmatter-only parent simulations, though neither contains such a satellite in the zoom-in runs we analyse here. Both hosts are presented in greater detail in Samuel et al. (2019).
} 


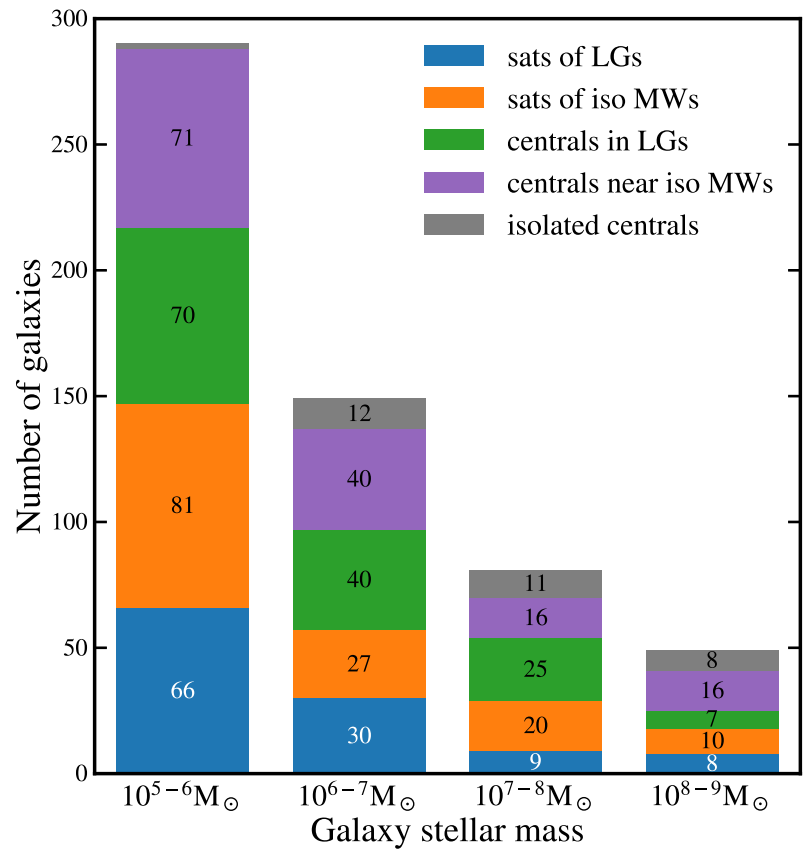

Figure 1. Our simulated sample, split into bins of stellar mass and separated by environment. It includes truly isolated ('isolated central'; gray) dwarf galaxies that are selected as the targets of a zoom-in simulation along with dwarfs that evolve alongside MW-mass hosts. We split the latter by their distance to the nearest such host at $z=0$ : satellites are defined as those with $d_{\text {host }} \leq 300 \mathrm{kpc}$ and centrals have $d_{\text {host }}>300-2000 \mathrm{kpc}$. We then further split these samples into 'satellites of LGs' (blue) and 'centrals in LGs' (green) taken from the two simulations targeting paired (LG-like) MW-mass hosts and 'satellites of isolated MWs' (orange) and 'centrals near isolated MWs' (purple) - taken from the eight simulations targeting individual MW-mass galaxies. Table 2 summarizes our environmental definitions. Throughout, we place a hard lower limit of 10 star particles per galaxy, but in practice, our lowest mass galaxies in our lowest resolution runs contain $\geq 14$ star particles.

the stellar mass converges to within 1 percent. ${ }^{6}$ We find that this method accurately and reliably separates real galaxies from transient alignments between sub-haloes and stars in the stellar haloes of the MW-mass hosts. Meanwhile, for the highly isolated dwarf central galaxies, which do not overlap with the extended stellar haloes that surround MW-mass hosts, we adopt all particles that are within the radius that contains 90 percent of the stellar mass within $20 \mathrm{kpc}$ of the galaxy centre. In all cases, we define $M_{*}$ as the sum of the masses of the member star particles and calculate SFHs using their formation times. Our smallest galaxies (with $M_{*} \simeq 10^{5} \mathrm{M}_{\odot}$ ) in our lowest resolution simulations (the isolated MW 'Latte' runs, with initial gas particle masses $m_{\mathrm{i}}=7100 \mathrm{M}_{\odot}$ ) therefore contain a minimum of 14 star particles, though stellar mass loss reduces the mass per particle such that the smallest galaxy we analyse actually contains 20 star particles. We discuss the potential for resolution artefacts, and their impact on our conclusions, in Section 3.3 and Appendix A.

${ }^{6}$ The catalogues therefore differ from those used in Garrison-Kimmel et al. 2019; most notably, those were based on haloes identified by the spherical overdensity-based AHF (Knollmann \& Knebe 2011). We have confirmed that the stellar mass functions and circular velocity profiles obtained via the new Rockstar catalogues are consistent with those of AHF.
Table 1. Simulations analysed in this work. Listed are the names of the zoom-in target halo, the stellar mass $\left(M_{*}\right)$ and halo mass $\left(M_{\text {halo }}\right)$ of that galaxy, the distance to $\left(d_{\text {nearest }}\right)$ and halo mass of $\left(M_{\text {halo }}^{\text {neart }}\right)$ the closest other halo with $M_{\text {halo }}>10^{11.5} \mathrm{M}_{\odot}$, the resolution of each simulation quantified by the initial baryonic particle mass $\left(m_{\mathrm{i}}\right)$, and the publication where each halo first appeared at the targeted resolution (see citations therein for earlier publications that feature lower resolution versions of most of the haloes).

\begin{tabular}{|c|c|c|c|c|c|c|}
\hline Simulation & $\begin{array}{c}M_{*} \\
\left(\mathrm{M}_{\odot}\right)\end{array}$ & $\begin{array}{c}M_{\text {halo }} \\
\left(10^{10} \mathrm{M}_{\odot}\right)\end{array}$ & $\begin{array}{l}d_{\text {nearest }} \\
(\mathrm{Mpc})\end{array}$ & $\begin{array}{c}M_{\text {halo }}^{\text {nearest }} \\
\left(10^{10} \mathrm{M}_{\odot}\right)\end{array}$ & $\begin{array}{c}m_{\mathrm{i}} \\
\left(\mathrm{M}_{\odot}\right)\end{array}$ & Ref \\
\hline \multicolumn{7}{|c|}{ LG hosts } \\
\hline Romeo & $7.36 \times 10^{10}$ & 132 & 0.84 & 110.5 & 3500 & A \\
\hline Juliet & $4.22 \times 10^{10}$ & 110 & 0.84 & 132.0 & 3500 & A \\
\hline Thelma & $7.92 \times 10^{10}$ & 143 & 0.92 & 115.3 & 4000 & A \\
\hline Louise & $2.85 \times 10^{10}$ & 115 & 0.92 & 143.3 & 4000 & A \\
\hline Romulus & $1.02 \times 10^{11}$ & 208 & 0.97 & 121.6 & 4000 & - \\
\hline Remus & $5.09 \times 10^{10}$ & 122 & 0.97 & 207.9 & 4000 & - \\
\hline \multicolumn{7}{|c|}{ Isolated MW hosts } \\
\hline $\mathrm{m} 12 \mathrm{~b}$ & $9.42 \times 10^{10}$ & 143 & 3.99 & 37.8 & 7100 & A \\
\hline $\mathrm{m} 12 \mathrm{C}$ & $6.45 \times 10^{10}$ & 135 & 4.68 & 267.7 & 7100 & A \\
\hline $\operatorname{m} 12 \mathrm{f}$ & $8.78 \times 10^{10}$ & 171 & 3.91 & 76.2 & 7100 & B \\
\hline $\mathrm{m} 12 i$ & $7 \times 10^{10}$ & 118 & 2.87 & 79.5 & 7100 & $\mathrm{C}$ \\
\hline $\mathrm{m} 12 \mathrm{~m}$ & $1.26 \times 10^{11}$ & 158 & 3.94 & 279.3 & 7100 & D \\
\hline $\mathrm{m} 12 \mathrm{r}$ & $1.88 \times 10^{10}$ & 110 & 6.70 & 1064.6 & 7100 & E \\
\hline $\mathrm{m} 12 \mathrm{w}$ & $6.29 \times 10^{10}$ & 108 & 2.63 & 88.2 & 7100 & E \\
\hline $\mathrm{m} 12 \mathrm{z}$ & $2.25 \times 10^{10}$ & 92.5 & 3.37 & 34.4 & 4200 & A \\
\hline \multicolumn{7}{|c|}{ Highly isolated dwarf centrals } \\
\hline $\mathrm{m} 10 \mathrm{~b}$ & $4.68 \times 10^{5}$ & 1.09 & 4.78 & 38.9 & 500 & $\mathrm{~F}$ \\
\hline $\mathrm{m} 10 \mathrm{C}$ & $5.76 \times 10^{5}$ & 1 & 6.36 & 276.9 & 500 & $\mathrm{~F}$ \\
\hline $\operatorname{miod}$ & $1.56 \times 10^{6}$ & 0.957 & 9.09 & 115.8 & 500 & $\mathrm{~F}$ \\
\hline $\mathrm{m} 10 \mathrm{e}$ & $1.99 \times 10^{6}$ & 1.17 & 6.47 & 44.9 & 500 & $\mathrm{~F}$ \\
\hline $\operatorname{m10f}$ & $4.2 \times 10^{6}$ & 0.943 & 4.83 & 55.0 & 500 & $\mathrm{~F}$ \\
\hline $\mathrm{m} 10 \mathrm{~g}$ & $5.74 \times 10^{6}$ & 0.846 & 3.93 & 349.9 & 500 & $\mathrm{~F}$ \\
\hline $\mathrm{m} 10 \mathrm{~h}$ & $7.95 \times 10^{6}$ & 1.45 & 9.94 & 85.5 & 500 & $\mathrm{~F}$ \\
\hline $\mathrm{m} 10 \mathrm{i}$ & $8.09 \times 10^{6}$ & 1.15 & 5.68 & 175.7 & 500 & $\mathrm{~F}$ \\
\hline $\mathrm{m} 10 \mathrm{j}$ & $9.83 \times 10^{6}$ & 1.2 & 3.60 & 58.5 & 500 & $\mathrm{~F}$ \\
\hline $\mathrm{m} 10 \mathrm{k}$ & $1.06 \times 10^{7}$ & 1.25 & 7.94 & 51.9 & 500 & $\mathrm{~F}$ \\
\hline m101 & $1.31 \times 10^{7}$ & 1.15 & 3.77 & 248.8 & 500 & F \\
\hline $\mathrm{m} 10 \mathrm{~m}$ & $1.47 \times 10^{7}$ & 1.24 & 6.72 & 106.1 & 500 & F \\
\hline m10xe_D & $3.8 \times 10^{6}$ & 1.04 & 3.79 & 53.4 & 4000 & G \\
\hline m10xe_A & $3.66 \times 10^{6}$ & 1.52 & 3.89 & 53.4 & 4000 & G \\
\hline m10xc_A & $8.85 \times 10^{6}$ & 0.97 & 5.60 & 38.3 & 4000 & G \\
\hline m10xe_B & $1.33 \times 10^{7}$ & 1.24 & 3.94 & 53.4 & 4000 & G \\
\hline m10xd_A & $1.48 \times 10^{7}$ & 3.29 & 2.94 & 78.2 & 4000 & G \\
\hline m10xe_c & $2.2 \times 10^{7}$ & 1.25 & 3.62 & 53.4 & 4000 & G \\
\hline m10xg_A & $1.96 \times 10^{7}$ & 1.89 & 6.22 & 85.2 & 4000 & G \\
\hline m10xb & $3.34 \times 10^{7}$ & 2.68 & 1.94 & 77.6 & 4000 & G \\
\hline m10xh_A & $5.48 \times 10^{7}$ & 1.77 & 3.58 & 124.3 & 4000 & G \\
\hline m10xd & $7.1 \times 10^{7}$ & 4.55 & 3.06 & 78.2 & 4000 & G \\
\hline m10xa & $8.07 \times 10^{7}$ & 2.16 & 6.18 & 343.2 & 4000 & G \\
\hline $\mathrm{m} 10 \mathrm{xc}$ & $1.21 \times 10^{8}$ & 3.93 & 5.55 & 38.3 & 4000 & G \\
\hline m10xf & $1.29 \times 10^{8}$ & 6.21 & 1.17 & 54.6 & 4000 & G \\
\hline m10xe & $3.32 \times 10^{8}$ & 5.36 & 4.28 & 53.4 & 4000 & G \\
\hline m10xi & $4.32 \times 10^{8}$ & 8.79 & 2.32 & 52.6 & 4000 & G \\
\hline m10xg & $4.59 \times 10^{8}$ & 7.18 & 6.01 & 85.2 & 4000 & G \\
\hline $\operatorname{m10x}$ & $5.23 \times 10^{8}$ & 9.19 & 3.09 & 486.7 & 4000 & G \\
\hline $\mathrm{m} 10 \mathrm{q}$ & $3.27 \times 10^{6}$ & 0.824 & 6.02 & 95.7 & 30 & $\mathrm{H}$ \\
\hline $\mathrm{m} 11 \mathrm{~h}$ & $1.43 \times 10^{8}$ & 18.6 & 4.10 & 146.0 & 880 & - \\
\hline $\mathrm{m} 11 \mathrm{~b}$ & $3.05 \times 10^{7}$ & 4.45 & 2.41 & 144.3 & 260 & - \\
\hline $\mathrm{m} 11 \mathrm{q}$ & $3.99 \times 10^{8}$ & 16.3 & 3.15 & 31.9 & 880 & I \\
\hline
\end{tabular}

Note: The references are A: Garrison-Kimmel et al. (2019), B: Garrison-Kimmel et al. (2017), C: Wetzel et al. (2016), D: Hopkins et al. (2018b), E: Samuel et al. (2019), F: Fitts et al. (2017), G: Graus et al. (2019), H: Wheeler et al. (2018), I: El-Badry et al. (2018a). 
Table 2. The five environments analysed in this work. The second column lists alternative terms for each environment sometimes adopted in the literature.

\begin{tabular}{lcl}
\hline Environment & Other terms & \\
\hline LG satellites & LG sub-haloes & Within 300 kpc of a MW-mass halo in an LG-like pair \\
LG centrals & Local field & More than 300 kpc from all MW-mass haloes, but within 2 Mpc of a MW-mass halo in an LG-like pair \\
& LG non-satellites & \\
& LG near-field & \\
Isolated MW satellites & Sub-haloes & Within 300 kpc of single (non-paired) MW-mass halo \\
Isolated MW centrals & Non-satellites & Between 300 kpc and 2 Mpc of a single MW-mass halo \\
Isolated centrals & Dwarf primaries & No MW-mass haloes within at least 2 Mpc \\
& field dwarfs & \\
& true-field dwarfs & \\
\hline
\end{tabular}

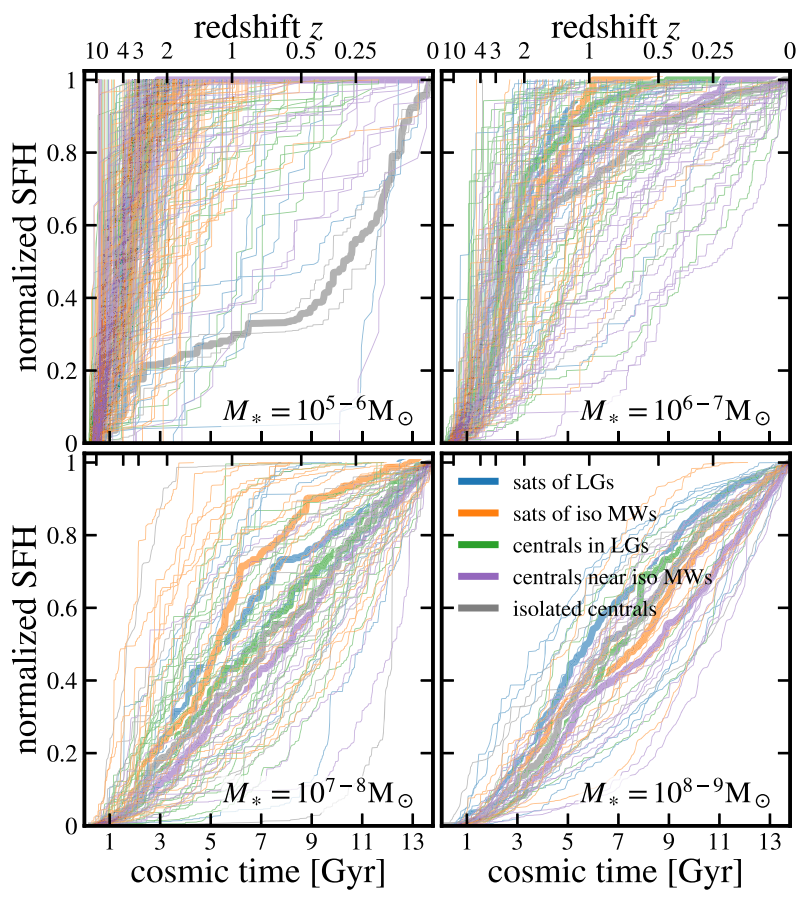

Figure 2. Cumulative archaeological SFHs of all dwarf galaxies in our sample, split into panels of stellar mass at $z=0$ and coloured by their environment. There is a clear trend across environments for higher mass galaxies to form a larger fraction of their stellar mass at later times, though the variety in the detailed SFHs at fixed $M_{*}$ is remarkable. The thick lines in the background plot the medians of each set of dwarf galaxies. As we discuss in Section 3.3, our lowest mass dwarf galaxies (with $M_{*} \leq 10^{6} \mathrm{M}_{\odot}$ ) around MW-mass host(s) may be subject to resolution effects that depress late-time star formation.

Throughout, we take dwarfs within $300 \mathrm{kpc}$ of a MW-mass host at $z=0$ as 'satellites' while dwarf galaxies more than $300 \mathrm{kpc}$ from a MW-mass galaxy are classified as 'centrals'. ${ }^{7}$ We further separate satellites into those of isolated MWs and of hosts in LG-like pairs, and split the centrals into those around isolated MWs, those in an LG-like environment, and highly isolated dwarf centrals that are the primary target of their zoom-in volumes. Table 2 summarizes the different environmental definitions and presents alternative names sometimes adopted in the literature.

\footnotetext{
${ }^{7}$ Our qualitative conclusions are insensitive to this choice: the median shapes
} change only slightly as we vary the satellite/central cut from $250-400 \mathrm{kpc}$.
For consistency with observations, we present archaeological SFHs throughout, calculated by taking the time that each star in the galaxy at $z=0$ formed. Therefore, some fraction of the stars included in the SFHs may have formed in an external galaxy and been brought in via mergers. Using the same Fitts et al. (2017) sample of isolated dwarf centrals as adopted here, Fitts et al. (2018) found that this fraction is typically small ( $<10$ per cent) for $M_{*} \lesssim 10^{7} \mathrm{M}_{\odot}$, and Anglés-Alcázar et al. (2017) found a similarly small fraction in a simulated FIRE galaxy with $M_{*}=1.4 \times 10^{9} \mathrm{M}_{\odot}$. While Deason, Wetzel \& Garrison-Kimmel (2014) used dark matter-only zoomin simulations (both of isolated MW-mass haloes and of LG-like pairs) to show that most dwarf haloes in LG-like environments have undergone a major merger at some point in their evolution (roughly $45-70$ per cent, with mergers more common among centrals and higher mass dwarf haloes) the vast majority of those mergers occur at cosmic time $t \lesssim 3 \mathrm{Gyr}$, before the majority of star formation in most of our sample (and also see Rodriguez-Gomez et al. 2016).

\section{RESULTS}

Fig. 2 summarizes the SFHs. Each panel plots the SFHs of the galaxies in our sample within a given decade of galaxy mass. The thin lines plot the individual galaxies, while the thick lines take the median of each environment.

Fig. 2 reveals two conclusions, which generally agree with previous results from both simulations and observations. First, for $M_{*} \lesssim 10^{8} \mathrm{M}_{\odot}$, there is an obvious trend with galaxy mass (across environments) where higher mass galaxies form a higher fraction of their stars at later times. Galaxies with $M_{*}=10^{5-6} \mathrm{M}_{\odot}$, which appear often to be dominated by starvation following reionization, typically quench (stop forming stars) by $t \sim 3 \mathrm{Gyr}^{8}{ }^{8}$ while galaxies with $M_{*} \geq 10^{8} \mathrm{M}_{\odot}$ almost universally continue to form stars to $z=0$. Secondly, even within a fixed mass bin (and in a fixed environment), there is a large degree of scatter in the SFHs. Even with this scatter, though, we find it exceptionally rare for galaxies to form their first stars at $t \gtrsim 1 \mathrm{Gyr}(z \lesssim 6)$. Only a few such galaxies exist in our sample, nearly all with $M_{*} \leq 10^{6} \mathrm{M}_{\odot}$. Moreover, only one galaxy (a low-mass central around an isolated MW-mass host) forms its first star after $t \simeq 4 \mathrm{Gyr}$, suggesting that galaxies of this

\footnotetext{
${ }^{8}$ We note that $\lesssim 10$ percent of satellites in this mass range have crossed $R_{200 \mathrm{~m}}$ of a MW-mass host by $t=3$, and that any trend between infall time and quenching time appears to break down at these masses. Both results strengthen the argument that non-environmental sources are most likely responsible for quenching these galaxies in our simulations.
} 

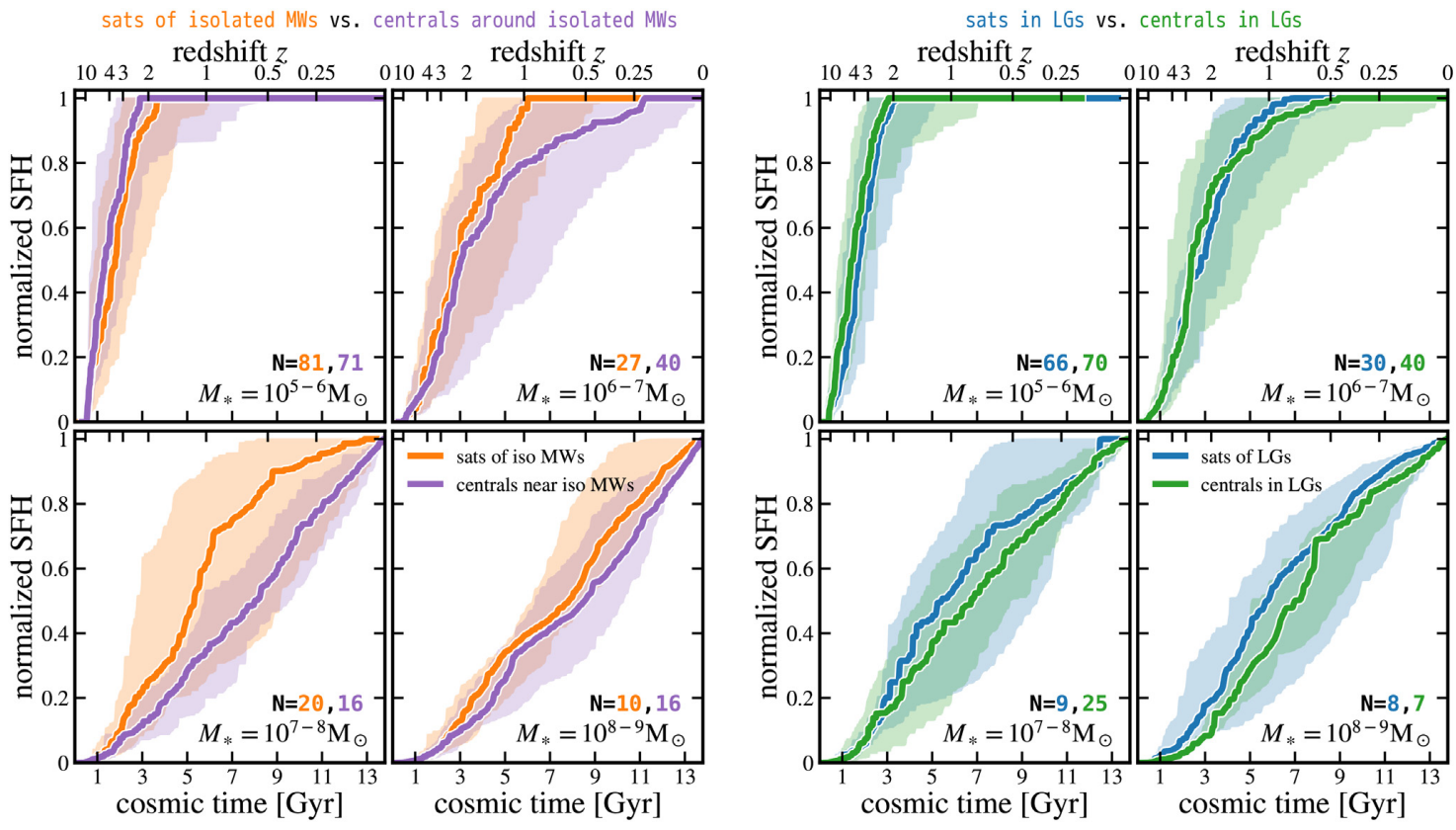

Figure 3. Median SFHs and 68 per cent scatter for dwarf galaxies binned by stellar mass and environment. The left-hand panel compares satellites $\left(d_{\text {host }}\right.$ $\leq 300 \mathrm{kpc}$ at $z=0)$ to centrals $\left(d_{\mathrm{host}}=300-2000 \mathrm{kpc}\right)$ around isolated MW-mass hosts. Centrals generally have more extended SFHs, consistent with a picture where satellites have their star formation quenched by interactions with the host. The right-hand panel shows the same comparison with dwarfs from the LG-like simulations. The satellite/central samples are relatively similar, suggesting that the LG environment impacts star formation in dwarf galaxies even beyond the virial radii of the hosts at a similar level to satellites inside the virial radius. The numbers in the lower right of each plot give the number of galaxies in each bin.

type are indeed very rare in $\Lambda \mathrm{CDM}$, in contrast with the predictions of warm DM models (Bozek et al. 2019).

\subsection{Environmental variations}

We now turn to the impact of environment on the shape of dwarf SFHs. We focus on comparing 'satellite versus central' and 'isolated MW versus LG-like pair versus isolated dwarf central,' but we will present statistics for all possible pairings below.

\subsubsection{Satellites versus centrals}

We begin with Fig. 3, which compares the SFHs of satellite galaxies to central galaxies. The left-hand panel selects only those objects that evolve around a single, isolated MW-mass host. As expected in environmental quenching models, satellites tend to reach a given fraction of their $z=0$ stellar mass at earlier times than centrals of a similar final mass. This is particularly evident for $M_{*}=10^{6}-10^{8} \mathrm{M}_{\odot}$, but the tail of late-time star formation in centrals is longer than that of the satellites even for $M_{*}=10^{5}-10^{6} \mathrm{M}_{\odot}$.

In contrast, the right-hand panel of Fig. 3 demonstrates that if such a distinction exists in the LG-like environments, it is strongly muted: satellites and centrals display far more similar behaviour overall with a smaller tail of late-time star formation for centrals with $M_{*} \leq 10^{7} \mathrm{M}_{\odot}$ than in the environments of isolated MWs.

\subsubsection{LGs versus isolated MWs versus the field}

Fig. 4 therefore compares the LG, isolated MW, and isolated dwarf samples, separating satellites and centrals. The median SFHs of the satellite populations (left-hand panel) of the LGs and isolated MWs are reasonably similar at most masses, though the LG satellites tend to form marginally earlier. The only deviation from this trend, and the mass range where the LG satellites differ the most from their isolated-MW counterparts, is for $M_{*}=10^{7}-10^{8} \mathrm{M}_{\odot}$. However, we caution that the LG-like simulations contain only nine satellites in that mass range.

The right-hand panel of Fig. 4 now compares the SFHs of dwarf central galaxies, including those around isolated MWs, those around LGs, and 'true field' dwarf centrals with no nearby MW-mass host. As expected from Fig. 3, the former two samples exhibit clear differences, particularly for $M_{*}=10^{6}-10^{8} \mathrm{M}_{\odot}$, where dwarf centrals in LGs tend to form their stars earlier than their counterparts around isolated MW-mass hosts. The offset is largest for $M_{*}=10^{6}$ $10^{7} \mathrm{M}_{\odot}$, where the medians are offset by nearly the full 68 per cent contours. We also emphasize that both the medians and scatters tend to shift relative to one another in the same sense. We examine the statistical significance of this result in Section 3.1.3 and argue in Section 3.3 that it is robust to resolution.

The truly isolated dwarf centrals appear to continue the trend between the number of nearby MW-mass hosts and the fraction of late-time star formation for $M_{*} \leq 10^{7} M_{*}$ : at fixed stellar mass, the isolated dwarf centrals tend to form later than those with MW-mass neighbours. While the difference in the lowest mass bin may be driven (at least partially) by resolution and our small sample size, the offset persists for $M_{*}=10^{6}-10^{7} \mathrm{M}_{\odot}$. Though the difference compared to centrals around isolated MWs is small, it is significant when compared with the centrals in LG-like environments. Therefore, the FIRE simulations predict that isolated dwarf galaxies cannot necessarily be fairly compared with dwarfs within $2 \mathrm{Mpc}$ of the LG - even those that are dwarf centrals - as the former will have formed more of their stars at late times. 

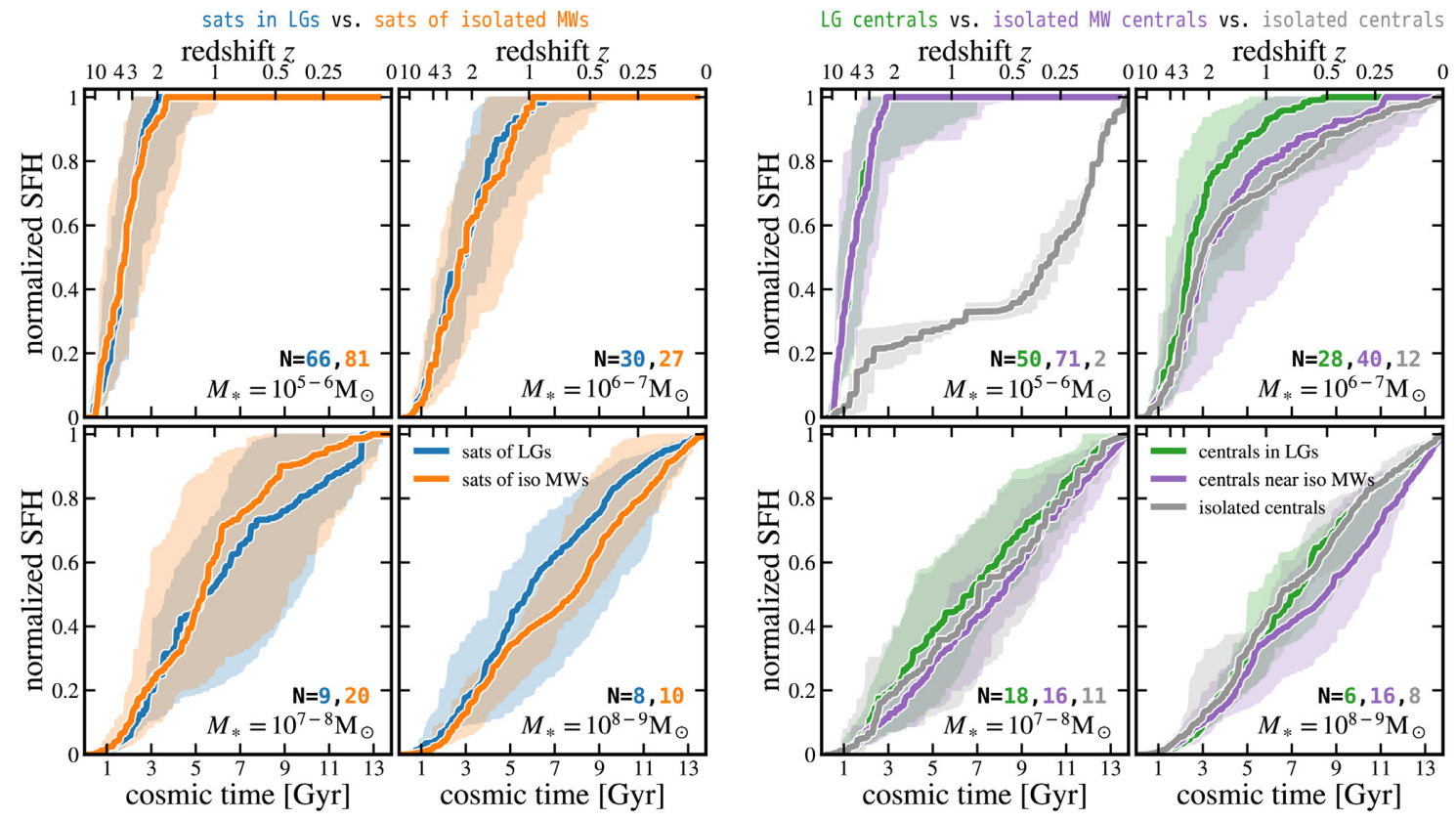

Figure 4. The SFHs of the satellites (left) and centrals (right) around isolated MWs compared to those in LG-like pairs and (in the right-hand panel) those far from any MW-mass host. The lines and shaded regions again indicate the median and 68 per cent contours. The satellite populations are similar at nearly all masses, though those in LG-like environments do tend to form marginally earlier at fixed $M_{*}$. The lone exception is for $M_{*}=10^{7}-10^{8} \mathrm{M}_{\odot}$, but the LG satellite sample includes only nine galaxies in that mass range. However, the SFHs of dwarf centrals exhibit stronger environmental trends: those around isolated MWs tend to form later than LG centrals at roughly all masses, and particularly for $M_{*}=10^{6}-10^{7} \mathrm{M}_{\odot}$. Though the shift in the medians is within the scatter, we note that the scatter also tends to shift in the same sense as the median. For $M_{*} \leq 10^{7} \mathrm{M}_{\odot}$, the highly isolated dwarf centrals have SFHs that are even more extended than the centrals around isolated MWs. Isolated centrals with $M_{*}=10^{5}-10^{6} \mathrm{M}_{\odot}$ display highly discrepant SFHs compared to dwarf centrals with at least one MW-mass host nearby, but we caution that the latter sample may be affected by resolution (see Section 3.3) and that there are only two galaxies in the former sample.

\subsubsection{Summary statistics of the SFHs}

To quantify these trends, we summarize the shapes of the SFHs via $t_{10}, t_{50}$, and $t_{90}$, the cosmic times when each galaxy reaches 10 per cent, 50 per cent, and 90 per cent of its $z=0$ stellar mass, respectively. Though we do not plot them, both $t_{10}$ and $t_{50}$ increase with $M_{*}$ (i.e. more massive dwarf galaxies have more extended SFHs), but do not display any clear systematic environmental variations within a given mass bin, particularly at low masses where the sample sizes are large. We also do not plot the fraction of 'intermediate-age' stars, which some previous studies on simulated SFHs have focused on (e.g. Benítez-Llambay et al. 2015; Digby et al. 2019). However, we note that our results for the fraction of stellar mass formed between $t=4$ and 8 Gyr in dwarf centrals with $M_{*} \geq 10^{7} \mathrm{M}_{\odot}$ is broadly consistent with the nearly constant 30 per cent found in the APOSTLE simulation (Digby et al. 2019). For $M_{*}=10^{6-7} \mathrm{M}_{\odot}$, however, that fraction ranges is slightly lower, ranging from 15 to 25 per cent.

The $t_{90}$ distributions, which are plotted in Fig. 5, display similar mass trends, but also exhibit clear environmental variations, consistent with our previous conclusions. As expected, these differences are strongest for $M_{*}=10^{6}-10^{7} \mathrm{M}_{\odot}$, but centrals around isolated MWs (purple lines) tend to have slightly later $t_{90}$ than either the LG centrals or the satellite populations at all masses. Taken together with the lack of a difference in the $t_{10}$ and $t_{50}$ distributions, Fig. 5 suggests that the main differences between the SFHs of dwarfs in different environments are in the amount of late-time star formation that occurs.

We quantify the statistical significance of the differences between the distributions of $t_{90}$ via the Anderson-Darling (AD) test
(Anderson \& Darling 1954) in Fig. 6. ${ }^{9}$ We highlight the $M_{*}=$ $10^{6}-10^{7} \mathrm{M}_{\odot}$ bin, which Fig. 5 shows has the largest environmental variations. Fig. 6 confirms that several of the differences in the $t_{90}$ distributions are statistically significant: in particular, both satellite populations quench earlier than both the dwarf centrals around single MWs and highly isolated dwarf centrals. LG dwarf centrals, meanwhile, sit in between the two extremes, but, from Fig. 5, far more closely resemble the satellite populations.

Though we do not plot them, we summarize the other mass bins here: for $M_{*}=10^{5}-10^{6} \mathrm{M}_{\odot}$, all of the $t_{90}$ distributions except that of the isolated dwarf centrals are broadly consistent with one another. The latter quenches much later, but our small sample of

\footnotetext{
${ }^{9}$ The AD test is an improved version of the well-known KolmogorovSmirnov (KS) test; we specifically adopt the $k$-sample generalization from Pettitt (1976, and also see Scholz \& Stephens 1987). The AD significance $p$ is the probability of finding differences in the distributions at least as extreme as those observed if the null hypothesis - that the two samples are drawn from the same underlying distribution - is correct. A low $p$-value therefore indicates a low probability of finding the observed differences, and therefore renders the null hypothesis unlikely. We chose a significance threshold that accounts for false positives by adopting the Bonferroni correction, which divides the nominal significance threshold $p \leq 0.05$ by the number of comparisons performed (Dunn 1961). We therefore consider cases with $p \leq$ 0.00125 as statistically significant and show the normalized $p$-value, $\hat{p}=$ $p / 0.00125$, such that $\hat{p} \leq 1$ indicates a statistically significant difference between two samples. We only show values for $p<0.25(\hat{p}<200)$. We also show $\hat{p}$ corresponding to the upper 95 per cent contour obtained by repeating the $\mathrm{AD}$ test on bootstrapped versions of the distributions, which we define as $\hat{p}_{95}$.
} 


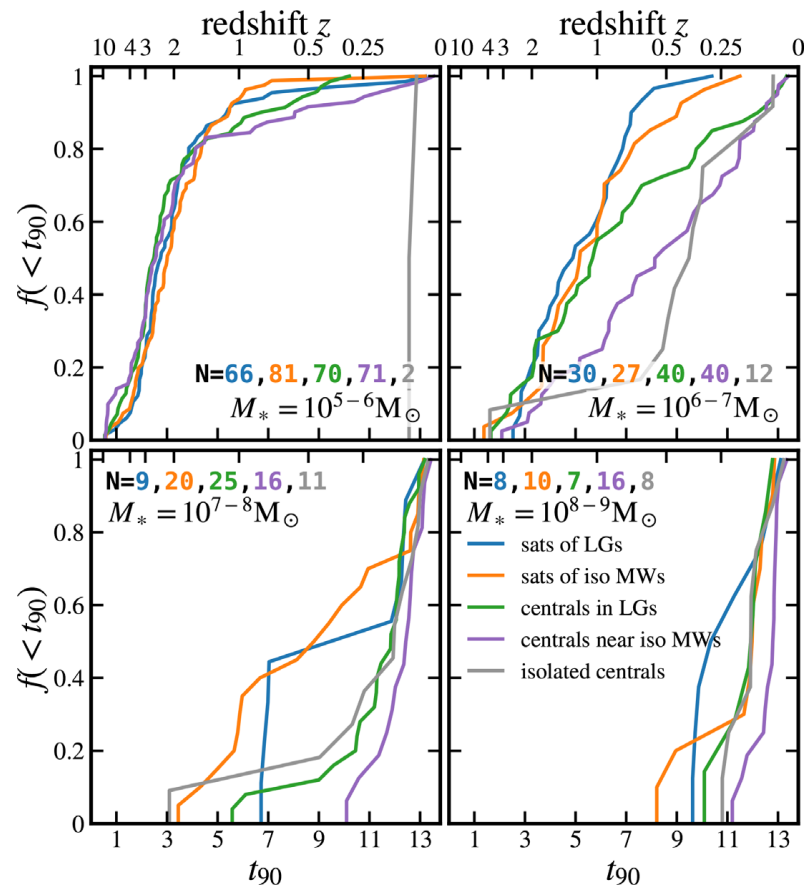

Figure 5. Distributions of the approximate quenching times of dwarf galaxies, quantified by the cosmic time when each galaxy reached 90 per cent $\left(t_{90}\right)$ of the stars in it at $z=0$. The distributions are computed by binning the dwarfs by stellar mass (panels) and environment (line colour; see legend). There is a strong trend for more massive dwarfs to quench later (if at all), regardless of environment. Within a given mass bin, satellite distributions are typically shifted to earlier times relative to centrals. As expected from previous figures, however, the differences between LG centrals and satellites is much smaller than that between centrals around isolated MWs and satellites. Fig. 6 quantifies the significance of these differences for $M_{*}=$ $10^{6}-10^{7} \mathrm{M}_{\odot}$.

isolated dwarfs with $M_{*}=10^{5}-10^{6} \mathrm{M}_{\odot}$ renders only the difference compared to LG centrals truly significant. There are no statistically significant offsets at higher masses, where larger samples are needed, but there are hints of differences between the LG centrals and isolated-MW centrals ( $\hat{p}=31.3$ and 20.4 for $M_{*}=10^{7-8}$ and $10^{8-9} \mathrm{M}_{\odot}$, respectively), between the satellites and centrals around isolated MWs ( $\hat{p}=2.5$ and 11.8 for the same bins), between the isolated-MW satellites and LG centrals at $10^{7-8} \mathrm{M}_{\odot}(\hat{p}=10.3)$, and between the LG satellites and isolated-MW centrals at $10^{8-9} \mathrm{M}_{\odot}$ $(\hat{p}=7.15)$.

We also do not plot the results of the AD test on the distributions of $t_{10}$ and $t_{50}$, which confirm that these statistics are largely insensitive to environment. The only significant variations arise in our lowest mass bin with the $t_{50}$ times of isolated dwarf centrals, which reach their half-mass times much later than the other four environments. However, many of the $t_{50}$ comparisons in this same mass bin yield $\hat{p}$ values that suggest changes with environment: only the 'LG satellite versus isolated-MW satellite' and 'LG central versus isolated-MW central' comparisons yield $\hat{p} \gtrsim 10$ for $M_{*}=10^{5}-10^{6} \mathrm{M}_{\odot}$.

\subsubsection{Why do dwarf centrals in LGs form their stars earlier?}

As the preceding sections showed, the SFHs of dwarf satellites in our sample appear independent of the larger scale environment (i.e. whether or not the host is in a LG-like pair), but the SFHs of our

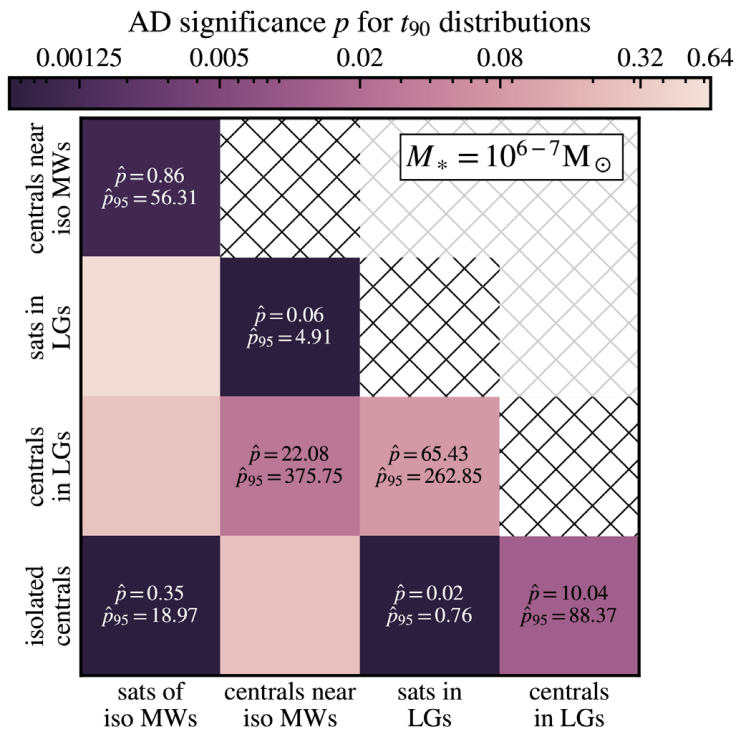

Figure 6. The statistical significance of the differences in the $t_{90}$ distributions (in the $10^{6}-10^{7} \mathrm{M}_{\odot}$ bin) between any pair of environments, quantified by the AD statistic (see footnote 9). $\hat{p} \leq 1$ indicates a statistically significant difference between the samples on the $x$ - and $y$-axes. Though it captures only a portion of the differences in the SFHs, the AD test shows that satellites of isolated MWs, LG satellites, and LG centrals, do not differ significantly from one another. However, all three of these populations differ from (quench earlier than) isolated dwarf centrals (bottom row) and centrals around isolated MWs. The difference between LG centrals and the latter two populations does not pass our corrected significance threshold, but the lack of a difference between satellites and centrals in LGs (especially compared to the significant offset between satellites and centrals around isolated MWs) is itself noteworthy. We discuss the results of the other mass bins and the other statistics in the text.

dwarf centrals in LGs exhibit less late-time star formation (at fixed final mass) than their counterparts around isolated MWs. In this section, we discuss possible explanations for the offset between the centrals - and the lack of an offset between the satellites - across the two environments.

We begin by exploring whether the offset in the SFHs can be explained via the dark matter accretion histories of the dwarf galaxies, which we quantify with $V_{\max }(t)$. Though it is much smaller than the offset between the SFHs, we do find that the dwarf centrals in the LGs and around isolated MWs display slightly different behaviours: while the median $V_{\max }(t)$ curve for centrals in LGs is slightly falling at late times, the corresponding curve in the isolated-MW runs is flat or slightly rising until $z=0$, particularly for the dwarf haloes hosting galaxies with $M_{*} \leq 10^{7} \mathrm{M}_{\odot}$. While the difference manifests at later times than the offset between the SFHs [e.g. for $M_{*}=10^{6-7} \mathrm{M}_{\odot}$, the median $V_{\max }(t)$ curves cross at $t \sim$ 7 Gyr while the median SFHs diverge at $t \sim 2 \mathrm{Gyr}$, the trend is in the direction expected if gravitational interactions are responsible for the offset in the SFHs. These interactions could be direct, e.g. in the form of an increased fraction of 'backsplash' haloes in LGs (centrals that were previously satellites; Teyssier, Johnston \& Kuhlen 2012; Garrison-Kimmel et al. 2014), or indirect, e.g. if the overall LG gravitational potential inhibits late-time accretion or if structure as a whole assembles earlier in LG environments. However, while the median $V_{\max }(t)$ for satellite galaxies is falling at late times in both environments (as expected), it falls slightly faster around the LG-like hosts; this is not reflected in the SFHs, which are 
roughly identical. Moreover, the change in $V_{\max }(t)$ moving from centrals to satellites (in either environment) is much larger than when comparing isolated-MW satellites (centrals) to LG satellites (centrals). We also find no evidence of an increased backsplash fraction: 25 percent of dwarf centrals with $M_{*}=10^{5}-10^{7}$ are backsplash systems, regardless of whether they are around a single MW or in an LG. ${ }^{10}$ Furthermore, removing backsplash systems from the comparison does not qualitatively change either the median $\mathrm{SFH}$ or the scatter about it. Therefore, while this explanation is qualitatively consistent and suggests that the difference may be due to a dynamical process, the fact that the trend is not reflected in the satellites and the relatively small size of the effect prohibits strong statements that the whole of the offset can be attributed to the dark matter accretion history or gravitational influences.

The offset in the SFHs of centrals could also be tied to the distribution of present-day distances from the nearest MW-mass host. The offsets between satellites and centrals suggest that quenching typically occurs later further from the host; therefore, if our LG centrals are typically further from their nearest host at $z=0$ than dwarf centrals around isolated MWs, the former may appear to quench earlier. We do find evidence, via $t_{90}$ versus $z=$ 0 distance, that quenching occurs (on average) later for galaxies further from a host today: fits to the data are nearly always rising with distance. ${ }^{11}$ However, dwarfs in LG-like environments quench (on average) earlier than their isolated-MW counterparts at roughly all $z=0$ distances. The difference is well within the scatter in all the mass bins, but it is most pronounced for $M_{*}=10^{6}-10^{7} \mathrm{M}_{\odot}$. Therefore, the offset cannot be attributed to the distribution of $z=$ 0 distances. Moreover, we find no clear evidence of the difference between LG dwarf centrals and dwarf centrals around isolated MWs disappearing at large distances.

Why, then, do the LG dwarfs (in both stars and DM) finish their formation or 'growth' phases earlier?

One possibility is that the LG regions are biased and systematically collapse earlier, compared to isolated MW or true-field regions. This is suggested by Fig. 7, which shows the LG hosts preferentially formed their stars 1-2 Gyr earlier than the isolated MW hosts. Similarly, comparing the halo growth histories shows the paired LG hosts reach half their $z=0$ mass 1 Gyr before the isolated MW-mass hosts. ${ }^{12}$ It is possible this is an artefact of small number statistics: while the number of individual LG dwarfs is large, the number of parent LG volumes is only three. In Garrison-Kimmel et al. (2018), we consider these and one additional LG volumes (4 total), albeit at lower resolution, and find the same average offset in host halo and star formation time in the additional volume as well. The CLUES project (Gottloeber et al. 2010) also found a similar effect in dark matter-only simulations of 3 LG analogues. However, in dark-matter-only simulations of 12 LG volumes, Garrison-Kimmel et al. (2014) found no significant difference in median LG halo formation times compared to isolated MW-mass systems. Moreover, we find no clear connection between the half-mass time of a MW-mass host and the quenching times

\footnotetext{
${ }^{10}$ This fraction drops sharply in the higher mass bins, which could be a physical effect if infall on to a MW-mass host quenches a galaxy. The trends with distance in this sample are also nearly identical between the isolated-MW and LG environments.

${ }^{11}$ The lone exception is in the lowest mass bin, where the fit to the LG dwarfs is very effectively flat.

${ }^{12}$ This may be a realization of the effect hypothesized by Gallart et al. (2015), who pointed out that higher density environments may assemble earlier and experience more star formation inducing mergers and interactions.
}

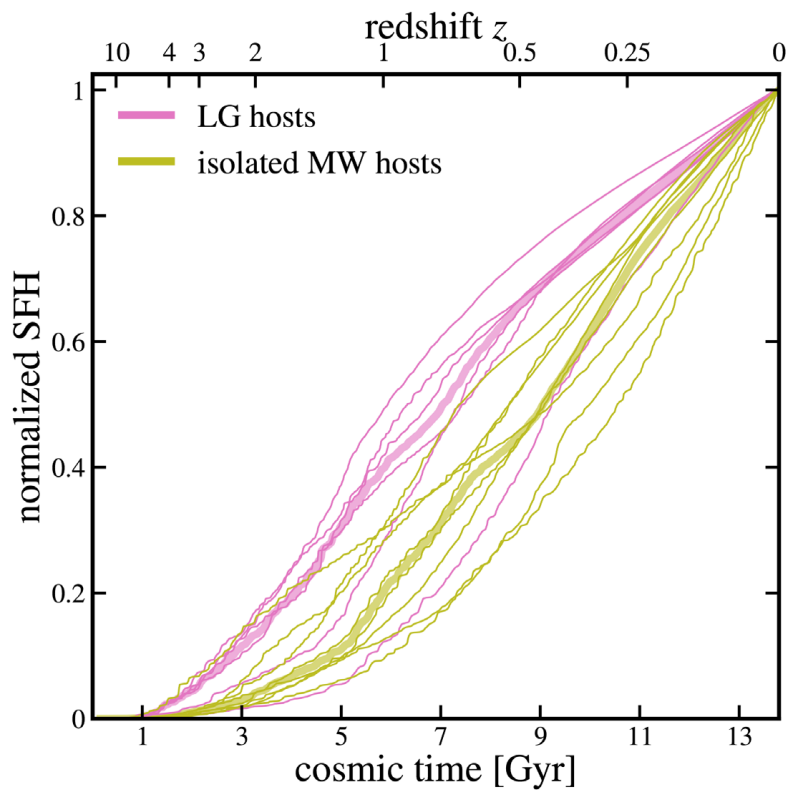

Figure 7. The archaeological SFHs of the MW-mass hosts in the simulations, separated into paired and isolated MWs. Three of the six paired hosts form half of their stars before any of the isolated MWs reach that milestone, with two of the remaining three almost exactly agreeing with the earliest forming isolated MW.

of the dwarf centrals that evolve nearby: dwarf centrals around the early-forming isolated MWs are not biased relative to those around the later forming isolated MWs, nor are the centrals near the late-forming LG-like host biased relative to those near the other five paired hosts. Clearly, a larger sample of LG volumes with baryonic physics and resolved dwarf populations is needed to robustly separate baryonic from dark matter effects, and (if the latter dominates) whether it is generic to all or most LG-type environments.

It is also possible that the offset in the SFHs is either driven by or exacerbated by purely baryonic processes. For example, a combination of ram pressure and turbulent viscous stripping can remove cold gas from dwarf satellites and shut down star formation, particularly if the hot gas in the host halo contains highdensity clumps (Fillingham et al. 2016) or if feedback within the satellite partially unbinds the cold gas (e.g. Hafen et al. 2019). These processes are, in principle, independent of any gravitational interactions. The 1-2 Mpc density fluctuation required to create an LG-like environment may cause the hot haloes of the hosts to merge and/or and extend further from the central galaxies. We do find evidence that the warm/hot gas $\left(T \geq 10^{5} \mathrm{~K}\right)$ extends further from the hosts in LG-like environments than the isolated MW-mass hosts. Even discounting gas that is nearer to the second host in the LG, five of the six LG-like hosts have warm gas at $\gtrsim 1 \mathrm{Mpc}$ while only two of the eight isolated MW-mass hosts display similar behaviour. While this intergalactic gas may not be dense enough or hot enough to actively strip dwarf galaxies, the comparatively high temperatures may inhibit infall of fresh gas (and therefore star formation). The relatively early SFHs of the hosts (Fig. 7) could also correlate with establishing hot haloes at earlier times or with supernovae-driven outflows extending further from the host at a given time - both effects would lead to more efficient quenching of nearby dwarf centrals. 

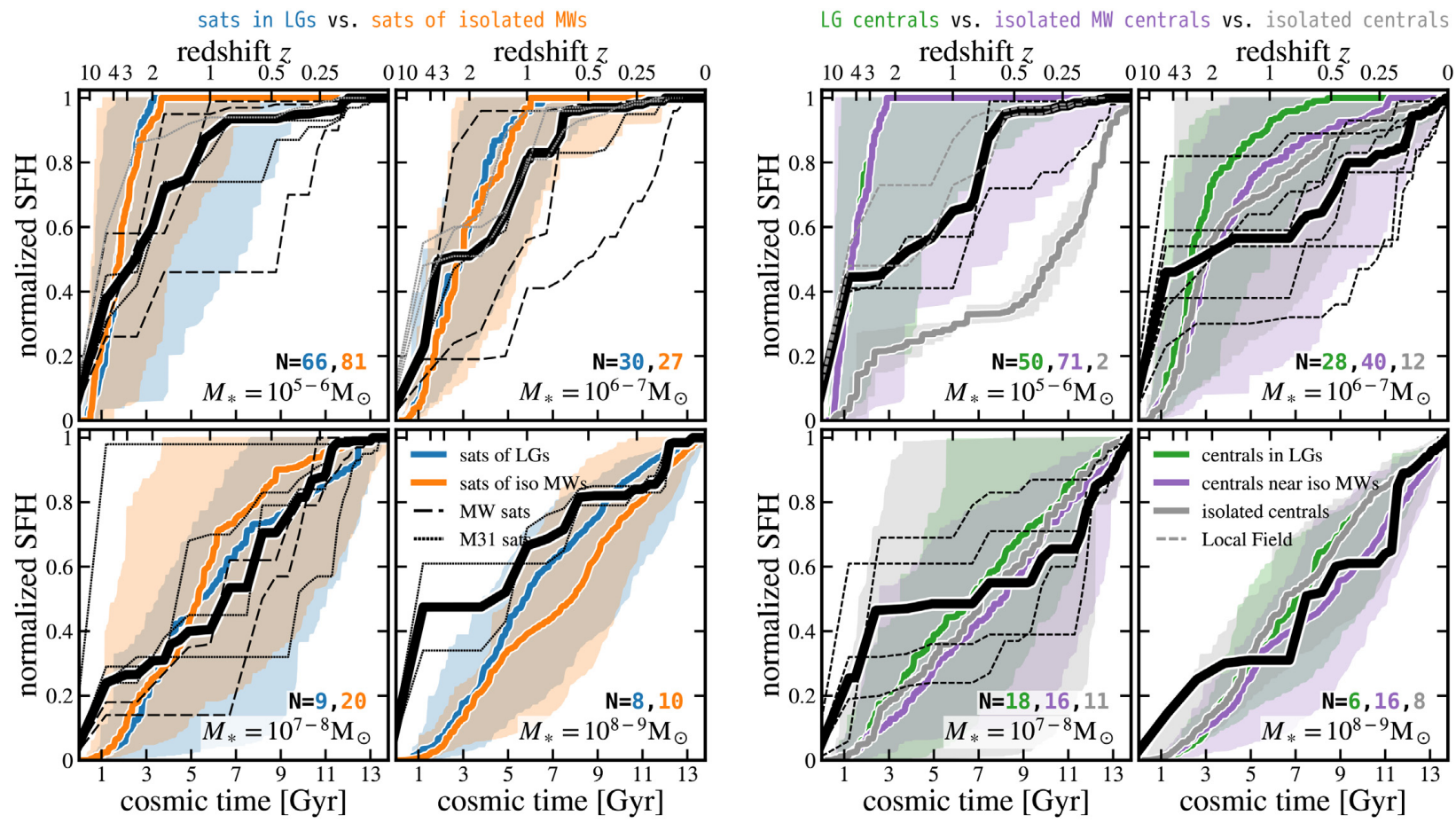

Figure 8. Comparing the SFHs of the simulated dwarf galaxies to observations of the dwarf satellites (left, with line styles according to the legend) and dwarf centrals (right) in the LG. Observed SFHs are taken from Weisz et al. (2014a, black lines) and Skillman et al. (2017, gray lines). ${ }^{13}$ Thick black lines plot the median of the observed galaxies in each bin. The simulated medians are identical to Fig. 4, but the shaded regions here indicate the 95 per cent contours. The simulations reproduce the trends with mass well, and the observations generally lie within the scatter from the simulations, but several of the lower mass galaxies in the LG have more late-time star formation than those in the FIRE simulations (more akin to the SFHs of our highly isolated dwarf centrals, though not as late forming). However, we caution that these galaxies are strongly influenced by, e.g. the redshift of reionization, which is relatively early in these simulations $\left(z_{\text {reion }} \simeq 10\right.$; see Appendix B).

\subsection{Comparisons with observations}

Fig. 8 repeats Fig. 4 by again plotting the SFHs of our simulated dwarf galaxies, but includes 95 percent contours (rather than 68 per cent) and adds the observed SFHs of MW/M31 satellites and of dwarf centrals in the LG. Observations are taken from Weisz et al. (2014a) and Skillman et al. (2017). ${ }^{13}$ The simulations generally reproduce the trends with mass well: for both satellites and centrals, the observed SFHs are typically within the 95 per cent scatter of the simulations. We also recover the observed trend (e.g. Wheeler et al. 2014; Weisz et al. 2015; Wetzel et al. 2015) wherein massive dwarf galaxies $\left(M_{*} \simeq 10^{8}-10^{9} \mathrm{M}_{\odot}\right)$ are nearly impervious to quenching: we find little differences between the SFHs of satellites and centrals in this mass range. However, the low-mass observed satellites have slightly more late-time star formation than the simulated counterparts, particularly for $M_{*}<10^{6} \mathrm{M}_{\odot}$. As we discuss below, this discrepancy may be at least partially due to resolution, and we note that the two higher resolution, highly isolated dwarf centrals in this mass bin have significant star formation at much later times so much so, in fact, that they underproduce the relative amount of early-time star formation compared to the observed dwarf centrals in the LG.

To better quantify these statements, we also perform the $\mathrm{AD}$ test between the observed $t_{90}$ distributions and their simulated analogues. For $M_{*} \leq 10^{7} \mathrm{M}_{\odot}$, the $\mathrm{AD}$ statistic indicates that the simulated and observed distributions are unlikely to be drawn from the same underlying set: the highest $p$ value obtained there (dwarf centrals around isolated MWs with $M_{*}=10^{6}-10^{7} \mathrm{M}_{\odot}$ ) is only 0.026. For $10^{7}-10^{8} \mathrm{M}_{\odot}$, satellites of isolated $\mathrm{MWs}$ have earlier quenching times on average than observations suggest $(p=0.023)$, as do centrals in LGs $(p=0.037)$. The sample size is very small above $M_{*}=10^{8} \mathrm{M}_{\odot}$ (particularly in the observations), leading to indeterminate comparisons with all of the $p$ values well above 0.25 .

\subsection{Caveats}

There are two main caveats to our results: the resolution of the simulations and the time at which the UV background (the 2011 December update of the Faucher-Giguère et al. 2009 model) reionizes the simulated volume. We discuss each of these here, though we defer a longer discussion of the former to Appendix A. Overall, we argue that neither of these caveats should impact our main results, particularly for $M_{*} \gtrsim 10^{6} \mathrm{M}_{\odot}$ (where the preceding figures suggest environmental differences exist).

\subsubsection{Resolution}

Perhaps the most obvious confounding variable in our analysis is the diversity of resolutions in the simulations: though all of our runs use identical physics, our isolated dwarf centrals vary from $m_{\mathrm{i}}=30-4000 \mathrm{M}_{\odot}$, the dwarfs in LG-like environments have $m_{\mathrm{i}}=$ 3500 or $4000 \mathrm{M}_{\odot}$, and those around isolated MW-mass galaxies have $m_{\mathrm{i}}=4200 \mathrm{M}_{\odot}(\mathrm{m} 12 \mathrm{z})$ or $7100 \mathrm{M}_{\odot}(\mathrm{m} 12 \mathrm{~b}-\mathrm{m} 12 \mathrm{w})$. The dependence of dwarf SFHs on resolution in the FIRE-2 simulations is discussed in Hopkins et al. (2018b, specifically in section 4.1.3 and fig. 8), but we review those results and discuss their impact on our conclusions here.

In brief, as $m_{\mathrm{i}}$ increases (i.e. resolution decreases), the individual bursts of star formation in a given dwarf become larger and more 
violent, as the smallest unit of stars that forms is tied to the gas particle mass. At the lowest resolutions, these artificial (i.e. numerical) bursts can become large enough that a single burst removes all of the gas from a dwarf, which can permanently shut off star formation in that dwarf and tends to reduce the amount of latetime star formation on average. None the less, total stellar masses remain remarkably consistent - typically to within 20 per cent, and within a factor of 3 even with only two star particles in the galaxy. Hopkins et al. (2018b) and Appendix A demonstrate that the SFH of a $10^{6} \mathrm{M}_{\odot}$ galaxy is reasonably well resolved at $m_{\mathrm{i}} \simeq 2100 \mathrm{M}_{\odot}$, though the $m_{\mathrm{i}}=30 \mathrm{M}_{\odot}$ simulation we use here is shifted to slightly later times. Therefore, the SFHs of the lower mass $\left(M_{*} \lesssim 10^{7} \mathrm{M}_{\odot}\right)$ dwarf galaxies taken from MW/LG environments, as well as those in the Graus et al. (2019) sample, may be somewhat underresolved.

Correcting for these artificially concentrated bursts should actually enhance the differences we find between LG-like environments and those of isolated MW-mass hosts, however. Dwarf galaxies in the LG-like simulations tend to form earlier than their analogues around isolated MW-mass hosts, even though the LG simulations are at slightly higher resolutions. Therefore, were the samples to be run at identical resolutions, we would expect to find an even larger difference between isolated MWs and LG-like environments, with the former exhibiting even more extended SFHs. We cannot, though, rule out the possibility that the offset between the highly isolated dwarf centrals and dwarf galaxies around MW-mass host(s), wherein the latter form earlier, is exaggerated by resolution. However, we show in Appendix A that the $m_{\mathrm{i}}=4000 \mathrm{M}_{\odot}$ Graus et al. (2019) galaxies form later than those in the $m_{\mathrm{i}}=500 \mathrm{M}_{\odot}$ Fitts et al. (2017) sample, suggesting that resolution effects are subdominant to mass trends and galaxy-to-galaxy scatter. We also note that correcting for these resolution trends would tend to shift the simulated SFHs more in line with the observations.

\subsubsection{Time of reionization}

By default (and in all the runs analysed here), the FIRE-2 simulations adopt the Faucher-Giguère et al. (2009) photoionizing background to capture the meta-galactic UV photons responsible for cosmic reionization. As mentioned above, that model was designed to match the WMAP-7 optical depth, corresponding to a reionization redshift of $z \simeq 10$ (Komatsu et al. 2011), in contrast with the most recent constraints on the mid-point of reionization from the Planck mission of $z=7.68 \pm 0.79$ (Planck Collaboration VI 2018). We emphasize, though, that all of the dwarf galaxies analysed here were simulated with an identical photoionizing background. Therefore, we expect that a later reionizing background would shift all of our SFHs (at a given mass) in the same manner; that is, the relative comparisons between the different environmental samples should be robust to the time of reionization.

However, this discussion ignores the effects of 'patchy' reionization. Both simulations (e.g. Trac \& Cen 2007; Ocvirk et al. 2016; Norman et al. 2018) and observations (e.g. Pentericci et al. 2014; Zheng et al. 2017) suggest some sections of the Universe may take until $z \sim 6.5$ to complete reionization. If reionization is highly patchy, and if the proto-MW galaxies contribute significantly to the local reionizing field, then we would naively expect that LG-like environments should reionize before those around isolated MWs, which should reionize before regions that host highly isolated dwarf galaxies (e.g. Alvarez et al. 2009; Lunnan et al. 2012). Therefore, at the masses where reionization interferes with star formation [roughly $M_{\text {halo }}\left(z=z_{\text {reion }}\right) \lesssim 10^{9} \mathrm{M}_{\odot}$; Dawoodbhoy et al. 2018], we would expect more early-time star formation in the isolated dwarf galaxies and less in those evolving in LG-like environments. As we discuss in Appendix B (and show explicitly in Fig. B1), a later $z_{\text {reion }}$ leads to a smaller fraction of late-time star formation, i.e. the normalized SFHs are shifted to earlier times. Consequently, patchy reionization could act to smear out the differences that we find between the three environments. However, reionization has the strongest impact on galaxies with $M_{*} \lesssim 10^{6} \mathrm{M}_{\odot}$ (e.g. Wheeler et al. 2015, 2018), and we find evidence of environmental variations at $M_{*}=10^{6}-10^{7} \mathrm{M}_{\odot}$. That is, the strongest differences exist for galaxies that should not be strongly impacted by the timing and patchiness of reionization.

Finally, in the process of preparing this manuscript, we discovered that an external heating term designed to mimic cosmic rays in the interstellar medium of MW-mass galaxies was being improperly applied to the intergalactic medium at extremely high redshift ( $z \gtrsim 20$; also see Su et al. 2018). Like the too-early reionization, this would act to suppress star formation at early times, such that a greater fraction of star formation instead occurs at later times. Correcting this mistake should therefore shift our SFHs to earlier times overall, since they represent the fractional mass formed by a given time. Internal testing indicates that this extraneous heating term has less than a 10 per cent impact on the shapes of SFHs for $M_{*} \sim 10^{6} \mathrm{M}_{\odot}$, with the strength of the effect scaling inversely with galaxy mass. Therefore, we expect this error to have a marginal impact for our three higher mass bins, and we again emphasize that, even at lower masses, all of our runs include this spurious heating term - correcting it should impact all of our SFHs in the same manner, and therefore only impact our conclusions with respect to the observations.

\section{CONCLUSIONS}

We have used a set of 500 dwarf galaxies - taken from FIRE-2 simulations of LG-like pairs of Milky Way-mass hosts separated by $\lesssim 1 \mathrm{Mpc}$, from isolated (single) MW-mass galaxies that are at least $\gtrsim 3 \mathrm{Mpc}$ from any other MW-mass systems, and from low-density regions that contain no MW-mass hosts in the simulation volume to explore how the shapes of dwarf SFHs vary with environment (both in terms of the number of MW-mass hosts nearby and the $z=$ 0 distance to the nearest such galaxy). Our main conclusions are:

(i) Even at fixed mass and environment, there is a large degree of scatter in SFHs, with the full sample often spanning $M_{*}(z) / M_{*}(z=$ $0) \simeq 0.2-1$ at fixed cosmic time.

(ii) None the less, the trends with mass in the median SFHs are robust: the fraction of stars formed at late times (roughly defined as cosmic time $t \geq 5 \mathrm{Gyr}$ ) increases with the $z=0$ stellar mass of the dwarf, nearly independent of environment (Figs 2 and 5).

(iii) The satellites (defined as galaxies within $300 \mathrm{kpc}$ of a MWmass host) of isolated MW-mass galaxies tend to form their stars earlier than equivalent dwarf centrals (non-satellite galaxies that are near a MW-mass host, but more than $300 \mathrm{kpc}$ away from that host today), consistent with a picture where interactions with MW-mass hosts inhibit star formation (Fig. 3).

(iv) Satellites in LG-like pairs have nearly identical SFHs to those of satellites around isolated MW-mass galaxies (Fig. 4).

(v) Dwarf central galaxies that evolve in LG-like environments have SFHs that are relatively similar to their satellite counterparts; that is, they contain older stars than central galaxies of similar masses around isolated MW-mass galaxies (Figs 3 and 4). 
(vi) Highly isolated dwarf galaxies with $M_{*} \leq 10^{7} \mathrm{M}_{\odot}$ form even later than the centrals around isolated MW-mass galaxies, suggesting a trend with the number of nearby MWs (Figs 4 and 5). However, the difference at $M_{*} \leq 10^{6} \mathrm{M}_{\odot}$ may be exaggerated by resolution, and the offset at $M_{*}=10^{6-7} \mathrm{M}_{\odot}$ relative to the centrals around isolated MW-mass hosts is not statistically significant, though the offset compared to the LG dwarf centrals is.

(vii) A statistical analysis of several summary statistics of each SFH (specifically, the time when each galaxy reaches 10 percent, 50 per cent, and 90 per cent of its mass at $z=0$ ), as a function of environment, indicates that - for $M_{*}=10^{6-7} \mathrm{M}_{\odot}$ - the satellites of isolated MWs and the dwarf galaxies in LGs (satellites or centrals) do not significantly differ from one another, though highly isolated dwarf centrals and the dwarf centrals around isolated (non-paired) MWs do differ strongly from the former three environments. The comparison between LG centrals and centrals around isolated MWs is inconclusive, but the $\mathrm{AD}$ test suggests that the former are more similar to the LG satellites than to the isolated-MW centrals (Fig. 6).

(viii) The simulations broadly reproduce the observed SFHs of both satellite and central dwarf galaxies in the LG. While they underproduce the amount of late-time star formation in our lowest mass dwarfs $\left(M_{*}=10^{5-6} \mathrm{M}_{\odot}\right)$, the disagreement is at least qualitatively consistent with resolution artefacts. However, there is a slight tension in that the observed SFHs of the LG centrals are slightly more consistent with the later forming centrals in simulations of isolated MWs, rather than with the (relatively) earlyforming centrals in simulations targeting LGs (Fig. 8). Moreover, the observed distributions of quenching times (quantified by the time at which each galaxy reaches 90 per cent of its $z=0$ stellar mass) are inconsistent with one another, particularly for $M_{*} \leq 10^{7} \mathrm{M}_{\odot}$ : the simulations quench too early on average.

Our results suggest that the MW-mass galaxies in the FIRE-2 simulations affect star formation in the dwarfs around them (though potentially indirectly), even when those dwarfs are not satellites at $z=0$. Therefore, caution should be taken when comparing simulations of dwarf galaxies that do not include any MW-mass galaxies, particularly if the properties under consideration are sensitive to the timing of star formation. Further work is required to solidify the significance of the differences (via increased sample sizes) and to identify their direct causes.

\section{ACKNOWLEDGEMENTS}

The authors thank Cameron Hummels, Peter Behroozi, Stephanie Tonnesen, and Zach Hafen for valuable discussions, Dan Weisz and Evan Skillman for making publicly available the observed SFHs, and Oliver Hahn and Peter Behroozi for making MUSIC and Rockstar public, respectively. This work was performed in part at the Aspen Center for Physics, which is supported by National Science Foundation grant PHY-1607611.

Support for SGK was provided by NASA through Einstein Postdoctoral Fellowship grant number PF5-160136 awarded by the Chandra X-ray Center, which is operated by the Smithsonian Astrophysical Observatory for NASA under contract NAS8-03060. SGK, AW, JSB, KEB, RES, MBK, and CAFG were supported by NASA through ATP \#80NSSC18K0562, 80NSSC18K1097, HST-GO12914, HST-GO-14734, HST-GO-14191, HST-AR-13888, HSTAR-13896, HST-AR-13921, HST-AR-14282, HST-AR-14554, HST-AR-15006, HST-AR-15057, JPL 1589742, NNX17AG29G, NNX15AB22G, and 17-ATP17-0067, many of which are awarded by STScI, which is operated by the Association of Universities for Research in Astronomy (AURA), Inc., under NASA contract NAS5-26555. SGK, PFH, JSB, AG, KEB, MBK, AF, TKC, DK, and CAFG were supported by the NSF through CAREER grant \#1455342, CAREER grant AST-1752913, CAREER grant AST1652522, Collaborative Research grant \#1715847, AST-1517226, AST-1517491, AST-1518291, AST-1715101, AST-1715216, and an NSF Graduate Research Fellowship. Additional support was provided by the Moore Center for Theoretical Cosmology and Physics at Caltech, the Alfred P. Sloan Research Fellowship, a Berkeley graduate fellowship, a Hellman award for graduate study, the Lee A. DuBridge Postdoctoral Scholarship in Astrophysics, the Swiss National Science Foundation (grant \#157591), and the Cottrell Scholarship Award from the Research Corporation for Science Advancement. The Flatiron Institute is supported by the Simons Foundation.

Numerical calculations were run on the Caltech compute cluster 'Wheeler,' allocations from XSEDE TG-AST130039 and PRAC NSF.1713353 supported by the NSF, NASA HEC SMD-16-7223, and SMD-16-7592, and High Performance Computing at Los Alamos National Labs. This work also made use of Astropy, a community-developed core PYTHON package for Astronomy (Astropy Collaboration et al. 2013; The Astropy Collaboration et al. 2018), matplotlib (Hunter 2007), numpy (van der Walt, Colbert \& Varoquaux 2011), scipy (Jones et al. 2001), ipython (Perez \& Granger 2007), yt (Turk et al. 2011), ytree (Smith \& Lang 2018), and NASA's Astrophysics Data System.

\section{REFERENCES}

Alvarez M. A., Busha M., Abel T., Wechsler R. H., 2009, ApJ, 703, L167 Anderson T. W., Darling D. A., 1954, J. Am. Stat. Assoc., 49, 765

Anglés-Alcázar D., Faucher-Giguère C.-A., Kereš D., Hopkins P. F., Quataert E., Murray N., 2017, MNRAS, 470, 4698

Astropy Collaboration et al., 2013, A\&A, 558, A33

Behroozi P. S., Wechsler R. H., Wu H.-Y., 2013, ApJ, 762, 109

Benítez-Llambay A., Navarro J. F., Abadi M. G., Gottlöber S., Yepes G., Hoffman Y., Steinmetz M., 2015, MNRAS, 450, 4207

Bland-Hawthorn J., Sutherland R., Webster D., 2015, ApJ, 807, 154

Boylan-Kolchin M., Weisz D. R., Johnson B. D., Bullock J. S., Conroy C., Fitts A., 2015, MNRAS, 453, 1503

Bozek B. et al., 2019, MNRAS, 483, 4086

Brooks A. M., Zolotov A., 2014, ApJ, 786, 87

Brown T. M. et al., 2014, Mem. Soc. Astron. Italiana, 85, 493

Buck T., Macciò A. V., Dutton A. A., Obreja A., Frings J., 2019, MNRAS, 483, 1314

Cole A. A. et al., 2007, ApJ, 659, L17

Cole A. A., Weisz D. R., Dolphin A. E., Skillman E. D., McConnachie A. W., Brooks A. M., Leaman R., 2014, ApJ, 795, 54

Colín P., Avila-Reese V., González-Samaniego A., Velázquez H., 2015, ApJ, 803,28

Dawoodbhoy T. et al., 2018, MNRAS, 480, 1740

Deason A., Wetzel A., Garrison-Kimmel S., 2014, ApJ, 794, 115

Digby R. et al., 2019, MNRAS, 485, 5423

Dunn O. J., 1961, J. Am. Stat. Assoc., 56, 52

Einasto J., Saar E., Kaasik A., Chernin A. D., 1974, Nature, 252, 111

El-Badry K. et al., 2018a, MNRAS, 473, 1930

El-Badry K. et al., 2018b, MNRAS, 477, 1536

Emerick A., Mac Low M.-M., Grcevich J., Gatto A., 2016, ApJ, 826, 148

Escala I. et al., 2018, MNRAS, 474, 2194

Fattahi A. et al., 2016, MNRAS, 457, 844

Faucher-Giguère C.-A., Lidz A., Zaldarriaga M., Hernquist L., 2009, ApJ, 703, 1416

Fillingham S. P., Cooper M. C., Wheeler C., Garrison-Kimmel S., BoylanKolchin M., Bullock J. S., 2015, MNRAS, 454, 2039 
Fillingham S. P., Cooper M. C., Pace A. B., Boylan-Kolchin M., Bullock J. S., Garrison-Kimmel S., Wheeler C., 2016, MNRAS, 463, 1916

Fillingham S. P., Cooper M. C., Boylan-Kolchin M., Bullock J. S., GarrisonKimmel S., Wheeler C., 2018, MNRAS, 477, 4491

Fillingham S. P. et al., 2019, preprint (arXiv:1906.04180)

Fitts A. et al., 2017, MNRAS, 471, 3547

Fitts A. et al., 2018, MNRAS, 479, 319

Gallart C. et al., 2015, ApJ, 811, L18

Garrison-Kimmel S., Boylan-Kolchin M., Bullock J. S., Lee K., 2014, MNRAS, 438, 2578

Garrison-Kimmel S. et al., 2017, MNRAS, 471, 1709

Garrison-Kimmel S. et al., 2018, MNRAS, 481, 4133

Garrison-Kimmel S. et al., 2019, MNRAS, 487, 1380

Geha M., Blanton M. R., Yan R., Tinker J. L., 2012, ApJ, 757, 85

Geha M. et al., 2017, ApJ, 847, 4

Gottloeber S., Hoffman Y., Yepes G., 2010, preprint (arXiv:1005.2687)

Governato F. et al., 2015, MNRAS, 448, 792

Grand R. J. J. et al., 2017, MNRAS, 467, 179

Graus A. S. et al., 2019, preprint (arXiv:1901.05487)

Gunn J. E., Gott J. R., III, 1972, ApJ, 176, 1

Gupta A., Mathur S., Krongold Y., Nicastro F., Galeazzi M., 2012, ApJ, 756, L8

Hafen Z. et al., 2019, MNRAS, 488, 1248

Hahn O., Abel T., 2011, MNRAS, 415, 2101

Hester J. A., 2006, ApJ, 647, 910

Hopkins P. F., 2015, MNRAS, 450, 53

Hopkins P. F., 2017, MNRAS, 466, 3387

Hopkins P. F., Narayanan D., Murray N., 2013, MNRAS, 432, 2647

Hopkins P. F., Kereš D., Oñorbe J., Faucher-Giguère C.-A., Quataert E., Murray N., Bullock J. S., 2014, MNRAS, 445, 581

Hopkins P. F., Grudic M. Y., Wetzel A. R., Keres D., Gaucher-Giguere C.-A., Ma X., Murray N., Butcher N., 2018a, preprint (arXiv:1811.12462)

Hopkins P. F. et al., 2018b, MNRAS, 480, 800

Hunter J. D., 2007, Comput. Sci. Eng., 9, 90

Jones E. et al., 2001, SciPy: Open source scientific tools for Python Available at: http://www.scipy.org/

Katz N., White S. D. M., 1993, ApJ, 412, 455

Kawata D., Mulchaey J. S., 2008, ApJ, 672, L103

Knollmann S. R., Knebe A., 2011, Astrophysics Source Code Library, record ascl:1102.009

Komatsu E. et al., 2011, ApJS, 192, 18

Kroupa P., 2001, MNRAS, 322, 231

Krumholz M. R., Gnedin N. Y., 2011, ApJ, 729, 36

Larson D. et al., 2011, ApJS, 192, 16

Larson R. B., Tinsley B. M., Caldwell C. N., 1980, ApJ, 237, 692

Lovell M. R. et al., 2017, MNRAS, 468, 4285

Lunnan R., Vogelsberger M., Frebel A., Hernquist L., Lidz A., BoylanKolchin M., 2012, ApJ, 746, 109

McConnachie A. W., 2012, AJ, 144, 4

Necib L., Lisanti M., Garrison-Kimmel S., Wetzel A., Sanderson R., Hopkins P. F., Faucher-Giguère C.-A., Kereš D., 2018, preprint (arXiv: 1810.12301)

Norman M. L., Chen P., Wise J. H., Xu H., 2018, ApJ, 867, 27

Nulsen P. E. J., 1982, MNRAS, 198, 1007

Ocvirk P. et al., 2016, MNRAS, 463, 1462

Oñorbe J., Garrison-Kimmel S., Maller A. H., Bullock J. S., Rocha M., Hahn O., 2014, MNRAS, 437, 1894

Oñorbe J., Boylan-Kolchin M., Bullock J. S., Hopkins P. F., Kereš D., Faucher-Giguère C.-A., Quataert E., Murray N., 2015, MNRAS, 454, 2092

Pearson S. et al., 2016, MNRAS, 459, 1827

Pentericci L. et al., 2014, ApJ, 793, 113

Perez F., Granger B. E., 2007, Comput. Sci. Eng., 9, 21

Pettitt A. N., 1976, Biometrika, 63, 161

Planck Collaboration XIII, 2016, A\&A, 594, A13

Planck Collaboration VI, 2018, preprint (arXiv:1807.06209)

Read J. I., Agertz O., Collins M. L. M., 2016, MNRAS, 459, 2573

Read J. I., Walker M. G., Steger P., 2019, MNRAS, 484, 1401
Ricotti M., Gnedin N. Y., Shull J. M., 2008, ApJ, 685, 21

Rocha M., Peter A. H. G., Bullock J., 2012, MNRAS, 425, 231

Rodriguez Wimberly M. K., Cooper M. C., Fillingham S. P., Boylan-Kolchin M., Bullock J. S., Garrison- Kimmel S., 2019, MNRAS, 483, 4031

Rodriguez-Gomez V. et al., 2016, MNRAS, 458, 2371

Samuel J. et al., 2019, preprint (arXiv:1904.11508)

Sanderson R. E. et al., 2018, ApJ, 869, 12

Sawala T. et al., 2016, MNRAS, 457, 1931

Scholz F. W., Stephens M. A., 1987, J. Am. Stat. Assoc., 82, 918

Simpson C. M., Grand R. J. J., Gómez F. A., Marinacci F., Pakmor R., Springel V., Campbell D. J. R., Frenk C. S., 2018, MNRAS, 478, 548

Skillman E. D. et al., 2017, ApJ, 837, 102

Smith B., Lang M., 2018, ytree: merger-tree toolkit. Available at: https: //doi.org/10.5281/zenodo.1174374

Spekkens K., Urbancic N., Mason B. S., Willman B., Aguirre J. E., 2014, ApJ, 795, L5

Su K.-Y., Hopkins P. F., Hayward C. C., Faucher-Giguère C.-A., Kereš D., Ma X., Robles V. H., 2017, MNRAS, 471, 144

Su K.-Y. et al., 2018, MNRAS, 480, 1666

Tanaka M., Chiba M., Hayashi K., Komiyama Y., Okamoto T., Cooper A. P., Okamoto S., Spitler L., 2018, ApJ, 865, 125

Teyssier M., Johnston K. V., Kuhlen M., 2012, MNRAS, 426, 1808

The Astropy Collaboration et al., 2018, AJ, 156, 123

Trac H., Cen R., 2007, ApJ, 671, 1

Turk M. J., Smith B. D., Oishi J. S., Skory S., Skillman S. W., Abel T., Norman M. L., 2011, ApJS, 192, 9

van der Walt S., Colbert S. C., Varoquaux G., 2011, Comput. Sci. Eng., 13, 22

Wadsley J. W., Stadel J., Quinn T., 2004, New A, 9, 137

Weisz D. R., Dolphin A. E., Skillman E. D., Holtzman J., Gilbert K. M., Dalcanton J. J., Williams B. F., 2014a, ApJ, 789, 147

Weisz D. R., Dolphin A. E., Skillman E. D., Holtzman J., Gilbert K. M., Dalcanton J. J., Williams B. F., 2014b, ApJ, 789, 148

Weisz D. R., Johnson B. D., Conroy C., 2014c, ApJ, 794, L3

Weisz D. R., Dolphin A. E., Skillman E. D., Holtzman J., Gilbert K. M., Dalcanton J. J., Williams B. F., 2015, ApJ, 804, 136

Wetzel A. R., Tinker J. L., Conroy C., van den Bosch F. C., 2013, MNRAS, 432, 336

Wetzel A. R., Tollerud E. J., Weisz D. R., 2015, ApJ, 808, L27

Wetzel A. R., Hopkins P. F., Kim J.-h., Faucher-Giguère C.-A., Kereš D., Quataert E., 2016, ApJ, 827, L23

Wheeler C., Phillips J. I., Cooper M. C., Boylan-Kolchin M., Bullock J. S., 2014, MNRAS, 442, 1396

Wheeler C., Oñorbe J., Bullock J. S., Boylan-Kolchin M., Elbert O. D., Garrison- Kimmel S., Hopkins P. F., Kereš D., 2015, MNRAS, 453, 1305

Wheeler C. et al., 2018, preprint (arXiv:1812.02749)

Wright A. C., Brooks A. M., Weisz D. R., Christensen C. R., 2019, MNRAS, 482,1176

Zheng Z.-Y. et al., 2017, ApJ, 842, L22

\section{APPENDIX A: RESOLUTION DEPENDENCE}

Section 3.3 discusses the expected impact of resolution on our results. We justify the conclusions we reach in that section here, but also refer the reader to section 4.1.3 of Hopkins et al. (2018b) for a longer discussion of the resolution elements required for the convergence of various galactic properties in the FIRE-2 simulations.

We begin with Fig. A1, which presents the SFH of a single, low-mass, isolated dwarf galaxy $(\mathrm{m} 10 \mathrm{q})$ as simulated at variety of resolutions. As we decrease the resolution (quantified by the initial gas particle mass $m_{\mathrm{i}}$ ) from the ultra-high resolution $m_{\mathrm{i}}=30 \mathrm{M}_{\odot}$ simulation that we analyse in the main text, the galaxy tends to form a greater fraction of its stars at later times. At the lowest resolution - which is even lower resolution than the simulations 


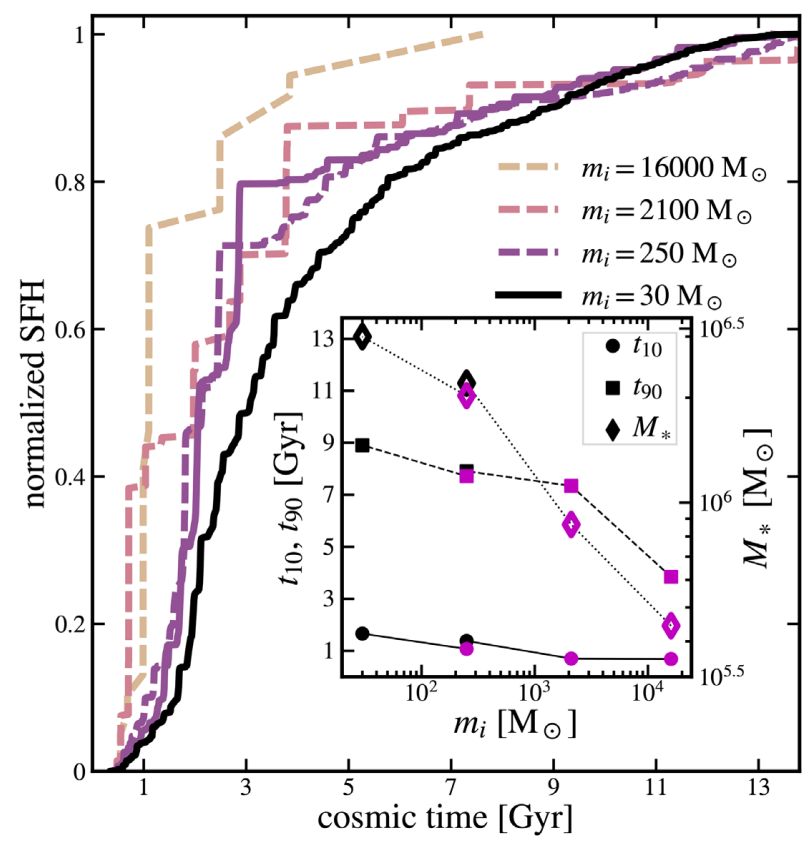

Figure A1. The SFH of a single isolated dwarf central galaxy (m10q, with $M_{*} \simeq 10^{6} \mathrm{M}_{\odot}$ ) in runs with initial gas particle masses $m_{\mathrm{i}}=30-$ $16000 \mathrm{M}_{\odot}$. For comparison, the LG and isolated MW simulations have resolutions $m_{\mathrm{i}}=3500-7100 \mathrm{M}_{\odot}$. The inset panel plots $t_{10}$ and $t_{90}$ for each run (squares and circles, left axis), as well as the stellar mass of the galaxy formed in each run (diamonds, right axis). Simulations that do not include subgrid metal diffusion are indicated with dashed lines and magenta symbols. m10q was simulated with $m_{\mathrm{i}}=250 \mathrm{M}_{\odot}$ both with and without this subgrid prescription; the SFHs from these two runs are nearly identical, indicating that metal diffusion has a negligible impact on the shape of the SFH. At lower resolutions (higher $m_{\mathrm{i}}$ ), the galaxy forms stars earlier. However, the $m_{\mathrm{i}}=2100 \mathrm{M}_{\odot}$ run agrees well with the run with $m_{\mathrm{i}}=250 \mathrm{M}_{\odot}$, with strongly divergent behaviour only appearing for $m_{\mathrm{i}}=16000 \mathrm{M}_{\odot}$ (more than twice the lowest resolution isolated-MW simulation). Moreover, these changes are also in line with the $M_{*}$ trends discussed in the main text: at lower resolution, the galaxy is also lower mass.

targeting isolated MW-mass hosts in the main text - the dwarf even artificially quenches at $t \simeq 7$ Gyr. However, the SFH is consistent between $m_{\mathrm{i}}=250 \mathrm{M}_{\odot}$ and $m_{\mathrm{i}}=2100 \mathrm{M}_{\odot}$, suggesting that the critical resolution at which we stop resolving the SFH of this dwarf (with $M_{*} \simeq 2 \times 10^{6} \mathrm{M}_{\odot}$ ) occurs between $m_{\mathrm{i}}=2100$ $16000 \mathrm{M}_{\odot}$. Unfortunately, our simulations that include MW-mass host(s) lie in between these two resolutions, though they do fall slightly closer to the lower edge of that range. Therefore, we acknowledge that our galaxies with $M_{*} \lesssim 10^{6} \mathrm{M}_{\odot}$ are somewhat underresolved and likely should have a higher fraction of late-time star formation. As we argue in Section 3.3, however, correcting for resolution effects should act to amplify the differences between the LG-like simulations and those of isolated MW-mass hosts, as the dwarf galaxies in the (slightly) higher resolution LGlike simulations already appear to have reached a given fraction of their $z=0$ stellar mass earlier. Moreover, the changes in Fig. A1 with resolution are also consistent with the mass trends identified in the main text: as $m_{\mathrm{i}}$ increases (resolution decreases), the galaxy forms earlier, but also reaches a smaller overall stellar mass.

Fig. A2 demonstrates that these mass trends, together with galaxy-by-galaxy variances, dominate over resolution effects (at our

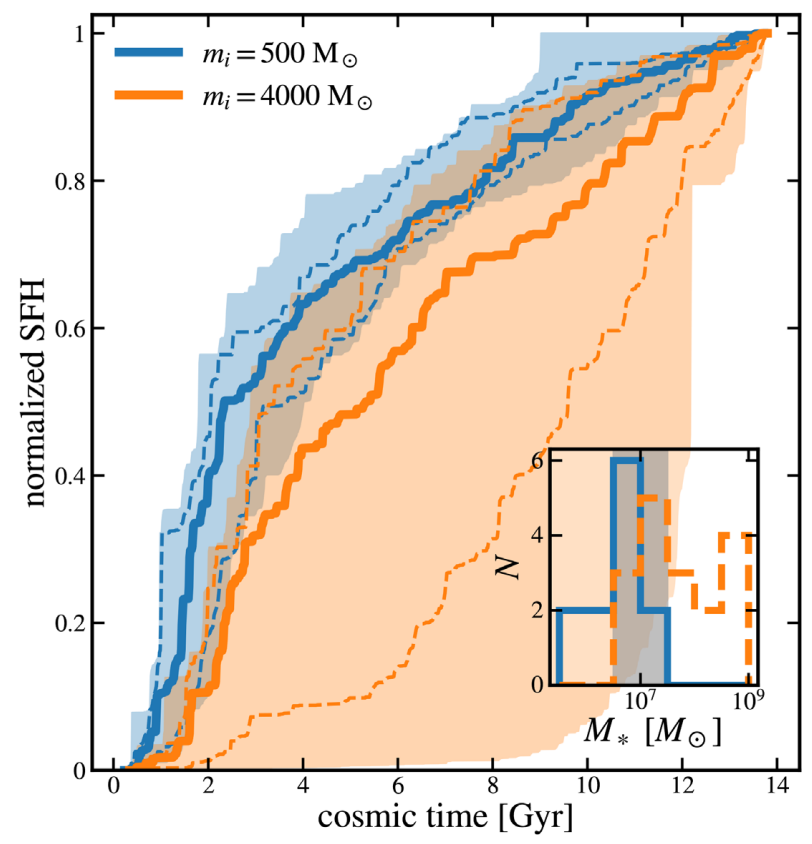

Figure A2. SFHs of highly isolated dwarf galaxies with stellar masses $M_{*}=10^{6.5}-10^{7.5} \mathrm{M}_{\odot}$; i.e. using a subset of the Fitts et al. (2017) and Graus et al. (2019) samples. Dashed lines indicate 68 percent of the sample, and the shaded areas indicate the full spreads. The lower resolution simulations tend to quench slightly later, in the opposite direction from the resolution trends in Fig. A1, but we point out from the inset panel that the lower resolution sample also tends towards higher masses, so the difference is in line with the stellar mass trends identified in the main text.

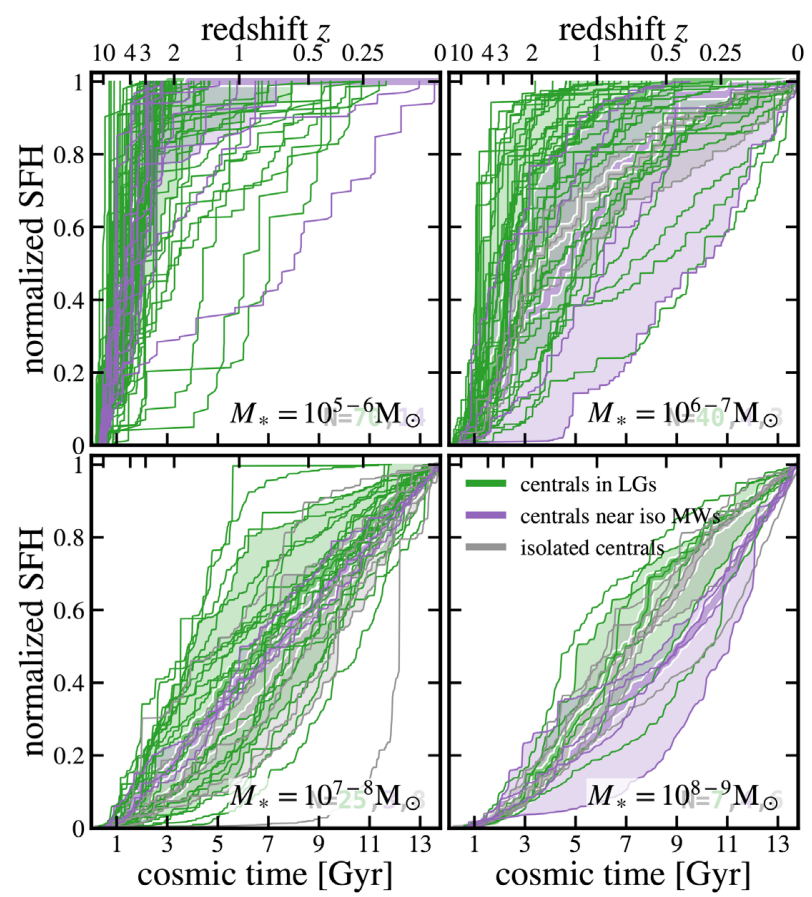

Figure A3. Comparing the SFHs of dwarf centrals from different environments at roughly fixed resolution $\left(m_{\mathrm{i}}=3500-4200 \mathrm{M}_{\odot}\right)$. Though the statistics are relatively poor in any individual bin (other than around the LGs), the conclusions identified above roughly hold true: dwarf centrals in LG-like environments form earlier than both the highly isolated centrals and those around a single MW. 
resolution). Here, we select highly isolated dwarf central galaxies with $M_{*}=10^{6.5}-10^{7.5} \mathrm{M}_{\odot}$ from two different sets of galaxies: the Fitts et al. (2017, in blue) and Graus et al. (2019, in orange) samples. That is, the two sets plotted in Fig. A2 are not the same galaxies at two different resolutions (unlike Fig. A1). Though the Graus et al. (2019) sample is at lower resolution, those galaxies form later than the dwarfs in the higher resolution Fitts et al. (2017) sample. As indicated by the inset panel, which plots the stellar masses of the two samples (with the shaded region representing the $M_{*}$ range of galaxies plotted in the main figure), the latter sample is at lower average mass (within this 1 dex mass bin).

As a final check on the impact of resolution, Fig. A3 plots the SFHs of dwarf centrals from simulations that have approximately identical resolution: the LG-like pairs $\left(m_{\mathrm{i}}=3500\right.$ and $\left.4000 \mathrm{M}_{\odot}\right)$, the Graus et al. (2019) sample $\left(m_{\mathrm{i}}=4,000 \mathrm{M}_{\odot}\right)$, and $\mathrm{m} 12 \mathrm{z}$ $\left(m_{\mathrm{i}}=4200 \mathrm{M}_{\odot}\right)$. Though the sample size is far too small to draw meaningful conclusions overall, the results identified above hold even in this limited set of simulations.

\section{APPENDIX B: EFFECTS OF A LATER REIONIZATION}

As discussed in Section 3.3, all of the simulations analysed in the main text adopt a reionizing background that completes reionization at $z \simeq 10$, while more recent observations suggest the Universe reionized quickly at $z \sim 7$. Fig. B1 demonstrates how changing $z_{\text {reion }}$ alters the SFHs of the satellites around a single (isolated) MW-mass host, m12i. As described in Section 3.3, a later $z_{\text {reion }}$ actually leads to more rapidly rising SFHs, as it allows for more star formation during the pre-reionization era, which reduces the relative fraction of stars that format late times. Furthermore, because a given galaxy tends to form more stars overall with later $z_{\text {reion }}$, some galaxies will shift to higher mass bins, further biasing the median SFH of the galaxies remaining in the lower mass bin towards earlier times (when normalizing the SFHs). We find qualitatively identical trends among the centrals around the m $12 i$, and when performing the same comparison with the dwarf galaxies around m12f (not plotted).

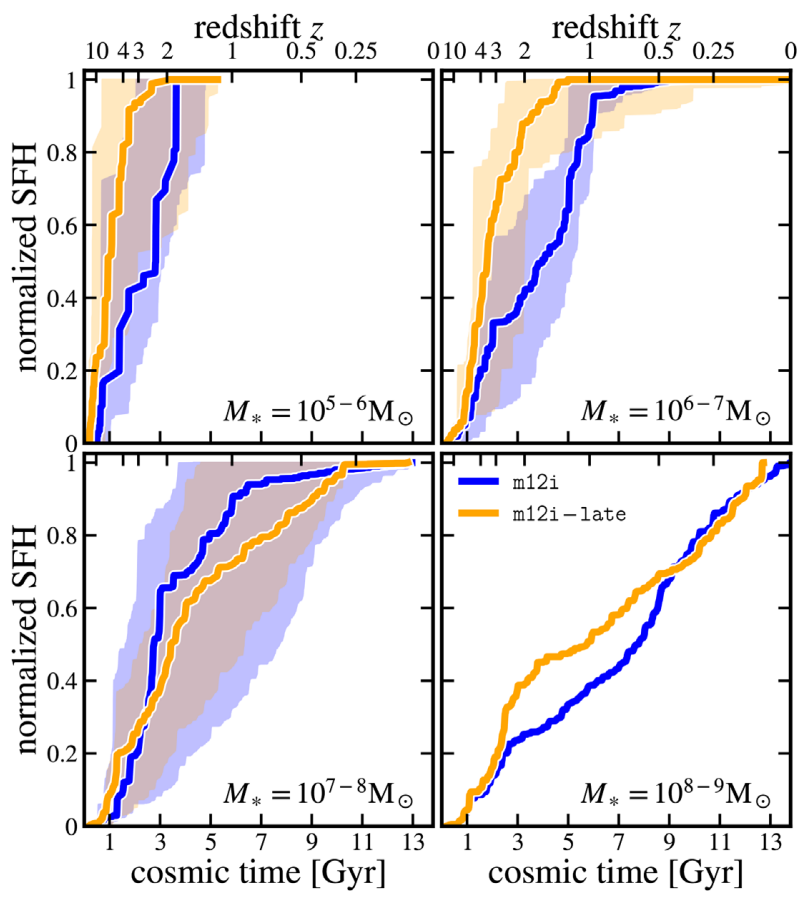

Figure B1. Comparing the SFHs of satellites around a single MW-mass host (m12i) run with the standard reionizing background (blue) and with a version of the Faucher-Giguère et al. (2009) UV background modified to produce a later reionization history that is consistent with the (Planck Collaboration VI 2018) electron scattering optical depth (orange). In this model, the hydrogen neutral fraction drops to 0.5 at $z \sim 7.8$. The thick lines take the median in each mass bin and the shaded regions indicate the full extent of the scatter. As discussed in the text, an earlier $z_{\text {reion }}$ tends to reduce the relative amount of early time star formation, as there is less time for stars to form before the background begins to play a role; the lines therefore tend to shift slightly to the left (more stars formed at earlier times) when moving to a background that reionizes the Universe at a later time.

This paper has been typeset from a $\mathrm{T}_{\mathrm{E}} \mathrm{X} / \mathrm{L}_{\mathrm{E}} \mathrm{X}$ file prepared by the author. 